\title{
Chemical abundances in Seyfert galaxies - VIII. Argon abundance estimates
}

\author{
A. F. Monteiro, ${ }^{1,2 \star}$ O. L. Dors, ${ }^{1}$ \\ ${ }^{1}$ Universidade do Vale do Paraíba. Av. Shishima Hifumi, 2911, CEP: 12244-000, São José dos Campos, SP, Brazil \\ ${ }^{2}$ Instituto Federal do Maranhão. Av. Newton Bello s/n, CEP: 65906-335, Imperatriz, MA, Brazil
}

Accepted XXX. Received YYY; in original form ZZZ

\begin{abstract}
For the first time, the argon abundance relative to hydrogen abundance $(\mathrm{Ar} / \mathrm{H})$ in the narrow line region of a sample of Seyfert 2 nuclei has been derived. In view of this, optical narrow emission line intensities of a sample of 64 local Seyfert 2 nuclei $(z<0.25)$ taken from Sloan Digital Sky Survey DR7 and measured by the MPA/JHU group were considered. We adopted the $T_{\mathrm{e}}$-method for AGNs, which is based on direct determination of the electron temperature, together with a grid of photoionization model results, built with the CLOUDY code, to obtain a method for the derivation of the $\mathrm{Ar} / \mathrm{H}$ abundance. We find that for a metallicity range of $0.2 \lesssim\left(\mathrm{Z} / \mathrm{Z}_{\odot}\right) \lesssim 2.0$, Seyfert 2 nuclei present $\mathrm{Ar} / \mathrm{H}$ abundance ranging from $\sim 0.1$ to $\sim 3$ times the argon solar value, adopting $\log (\mathrm{O} / \mathrm{H})_{\odot}=-3.31$ and $\log (\mathrm{Ar} / \mathrm{H})_{\odot}=-5.60$. These range of values correspond to $8.0 \lesssim(12+\log (\mathrm{O} / \mathrm{H}) \lesssim 9.0$ and $5.4 \lesssim(12+\log (\mathrm{Ar} / \mathrm{H}) \lesssim 6.9$, respectively. The range of $\mathrm{Ar} / \mathrm{H}$ and $\mathrm{Ar} / \mathrm{O}$ abundance values obtained from our sample are in consonance with estimations from extrapolations of the radial abundance gradients to the central parts of the disk for four spiral galaxies. We combined our abundance results with estimates obtained from a sample of $\mathrm{H}$ II galaxies, which were taken from the literature, and found that the $\mathrm{Ar} / \mathrm{O}$ abundance ratio decreases slightly as the $\mathrm{O} / \mathrm{H}$ abundance increases.
\end{abstract}

Key words: galaxies: Seyfert - galaxies: active - galaxies: abundances -ISM: abundances -galaxies: evolution -galaxies: nuclei

\section{INTRODUCTION}

Active Galactic Nuclei (AGNs) present strong metal emission-lines in their optical spectra, which when combined with hydrogen recombination lines, make it possible to estimate the abundance of heavy elements and the metallicity in the gas phase of these objects. AGNs play an essential role in chemical abundance studies of nearby objects and of the early stages of galaxy formation due to the aforementioned feature and to their high luminosity.

Among the heavy elements, oxygen presents strong emission lines (i.e. $[\mathrm{O}$ II] $\lambda 3726, \lambda 3729$; [O III] $\lambda 5007$ ) emitted by its most abundant ions $\left(\mathrm{O}^{+}, \mathrm{O}^{2+}\right)$ in the optical spectrum of gaseous nebulae ( $\mathrm{H}$ II regions, Planetary Nebulae) and AGNs (e.g. Koski 1978; van Zee et al. 1998; Kennicutt et al. 2003; Maciel et al. 2007; Dopita et al. 2015; Flury \& Moran 2020; Dors et al. 2020a). Therefore, the total metallicity $(Z)$ of the gas phase from emission lines emitter objects is commonly traced by the oxygen abundance relative to hy-

* E-mail: adriano.santos@ifma.edu.br drogen (O/H, e.g. McGaugh 1991; Yates et al. 2012; Kewley et al. 2019). Other elements such as the noble gases (e.g. $\mathrm{Ne}, \mathrm{Ar}$ ) present emission-lines in the optical spectrum emitted by only few of their ions (e.g. $\mathrm{Ne}^{2+}, \mathrm{Ar}^{2+}$ ), which make it necessary to apply Ionization Correction Factors (ICFs) proposed by Peimbert \& Costero (1969) (see Stasińska 2002; Delgado-Inglada et al. 2014; Dors et al. 2013, 2016) in order to account for the unobserved ions in the estimation of the total abundance. The use of ICFs can introduce uncertainties in order of $20 \%$ (e.g. Henry et al. 1996; Alexander \& Balick 1997; Croxall et al. 2016) in the resulting total abundance (for a detailed discussion on ICF uncertainties see Delgado-Inglada et al. 2014). Despite this drawback, noble gases can be used to derive the metallicity with some advantages over oxygen, as they are also useful elements for determining constraints in stellar nucleosynthesis studies. Noble gas atoms can not combine in molecules formation and they can not be trapped in dust grains due to their quantum configuration, unlike the oxygen which is depleted onto dust in order of 0.1 dex (e.g. Izotov et al. 2006; Pilyugin et al. 2007).

The $T_{\mathrm{e}}$-method, which is based on direct estimation of 
the electron temperature, is widely used in the literature as the most reliable approach for determining the chemical abundance of heavy metals in gaseous nebulae (for a review see Peimbert et al. 2017; Pérez-Montero 2017). The $T_{\mathrm{e}}$-method has been bolstered by the consonance between $\mathrm{O} / \mathrm{H}$ abundance estimates in $\mathrm{H}$ II regions in the solar vicinity and those obtained from observations of the weak interstellar O I $\lambda 1356$ line towards stars (see Pilyugin 2003 and references therein). Moreover, in the Milky Way and in nearby galaxies, good agreement between $\mathrm{O} / \mathrm{H}$ abundance estimates in $\mathrm{H}$ II regions and in B-type stars has recently been derived (e.g. Toribio San Cipriano et al. 2017). In this regard, abundance determinations of heavy metals (O, N, S, Ar, etc.) based on the $T_{\mathrm{e}}$-method have been carried out in thousands of star-forming regions (SFs; i.e. $\mathrm{H}$ in regions, $\mathrm{H}$ in galaxies) at the local universe and for certain objects at high redshifts over decades (see Dors et al. 2020a and reference therein).

Unfortunately, the situation is opposite for AGNs, where, except from oxygen, the majority of the abundances for other elements are not available in the literature. In fact, the most complete metal abundance determinations based on $T_{\mathrm{e}}$-method was carried out by Osterbrock \& Miller (1975) for Cygnus A $(z=0.05607)$, who derived the O, N, Ne, S, and Fe abundances in relation to hydrogen. After this pioneering work some few studies have applied the $T_{\mathrm{e}}$-method to abundance estimations in AGNs, however, in most cases, only oxygen abundance determinations have been derived (e.g. Alloin et al. 1992; Izotov \& Thuan 2008; Revalski et al. 2018a,b, 2021; Dors et al. 2015, 2020b,a). Recently, Flury \& Moran (2020), adopting a methodology based on a reverseengineering of the $T_{\mathrm{e}}$-method, derived the first $(\mathrm{N} / \mathrm{O})-(\mathrm{O} / \mathrm{H})$ relation for AGNs. Although studies relied on photoionization models have been applied to derive metal abundance in AGNs (e.g. Stasińska 1984; Ferland \& Osterbrock 1986; Storchi-Bergmann et al. 1998a; Groves et al. 2006; Feltre et al. 2016; Castro et al. 2017; Pérez-Montero et al. 2019; Thomas et al. 2019; Carvalho et al. 2020; Dors et al. 2021; Pérez-Díaz et al. 2021), most of them have produced only estimations for $\mathrm{O} / \mathrm{H}$ or metallicity. Since the electron temperatures throughout the emission nebula are computed by thermal balancing (see the seminal paper by Williams 1967), the abundances of the most important elements are used as input parameters in photoionization models. All the lines, even if not observed, contribute to the gas cooling rate. In most of the papers which describe the results obtained by using photoionization models, the element relative abundances to $\mathrm{H}$, which were not found to be particularly different from the solar ones, are unfortunately not published in the literature.

The use of photoionization model to derive abundance of different elements other than the oxygen (e.g. N, S, Ar) is (relatively) difficult, hence, it is necessary to find a solution for the electron temperature (or for $\mathrm{O} / \mathrm{H}$, the main cooler element) and for the ionization degree of the gas. Afterwards, the lines of the element under study must be adjusted in order to obtain its abundance (see, for instance, PérezMontero \& Díaz 2007; Pérez-Montero et al. 2010; Contini 2017; Congiu et al. 2017; Dors et al. 2017; Polles et al. 2019; Dors et al. 2021). This procedure can produce a degeneracy among nebular parameters, resulting in somewhat uncertain elemental abundances (Morisset et al. 2016; Morisset 2018). In this sense, the use of the $T_{\mathrm{e}}$-method produces more ex- act elemental abundance values in comparison with those estimated through photoionization models.

In particular, the argon abundance determination in AGNs is very important in the study of galaxy evolution and stellar nucleosynthesis, since the stellar production and later ejection of this element to the Interstellar Medium (ISM) in the high metallicity regime can be accessed. The stellar nucleosynthesis theory predicts a primary origin for oxygen and argon (also for sulphur and neon) which are predominantly produced on relatively short timescales by corecollapse supernovae (SNe; massive stars) explosions (e.g. Woosley \& Weaver 1995). Thus, assuming a universal Initial Mass Function (IMF) ${ }^{1}$, there is expectation for a relatively constant value of the $\mathrm{Ar} / \mathrm{O}$ abundance ratio with the $\mathrm{O} / \mathrm{H}$ (or metallicity) variation, as derived by several authors in chemical abundance studies of SFs (e.g. Thuan et al. 1995; Izotov \& Thuan 1999; van Zee \& Haynes 2006; Guseva et al. 2011; van Zee \& Haynes 2006). However, some authors have found different behaviour of $\mathrm{Ar} / \mathrm{O}$ with $\mathrm{O} / \mathrm{H}$. For example, Izotov et al. (2006), who used a large sample of star-forming galaxies whose observational data were taken from SDSS-DR3 (Abazajian et al. 2005), found that $\mathrm{Ar} / \mathrm{O}$ abundance ratio decreases by 0.15 dex with the increase of $\mathrm{O} / \mathrm{H}$ for the range $7.1 \lesssim 12+\log (\mathrm{O} / \mathrm{H}) \lesssim 8.5$ (see also Pérez-Montero et al. 2007). On the other $\tilde{\text { hand, }}$ recent results from the CHAOS project (Berg et al. 2015) derived by Berg et al. (2020), who applied the $T_{\mathrm{e}}$-method to 190 individual $\mathrm{H}$ II regions located in nearby galaxies, found $\mathrm{Ar} / \mathrm{O}$ about constant and similar to the solar value for the range $8.3 \lesssim 12+\log (\mathrm{O} / \mathrm{H}) \lesssim 9.0$, and a high decrease of this abundance ratio for the very low abundance regime. Finally, Kennicutt et al. (2003) hinted that the Ar/O abundance decreases at high metallicity $(8.5 \lesssim 12+\log (\mathrm{O} / \mathrm{H}) \lesssim 8.7)$ in the M 101 spiral galaxy.

For the very high metallicity regime $(12+$ $\log (\mathrm{O} / \mathrm{H}) \gtrsim 8.8)$, the behaviour of $\mathrm{Ar} / \mathrm{O}$ with $\mathrm{O} / \mathrm{H}$ is poorly known as well as its abundance in AGNs. Recently, Dors et al. (2020a) adapted the $T_{\mathrm{e}}$-method to chemical abundance studies of AGNs, which made it possible to obtain direct $\mathrm{O} / \mathrm{H}$ estimates up-to the very high metallicity regime, i.e. $12+\log (\mathrm{O} / \mathrm{H}) \approx 9.3$, an abundance value which is about 0.3 dex higher than the maximum value obtained for SFs (Pilyugin et al. 2007; Berg et al. 2020). Therefore, abundance studies in AGNs through the $T_{\mathrm{e}}$-method allow for the calculations of reliable $\mathrm{Ar} / \mathrm{H}$ abundances in this class of object and further investigating the $\mathrm{Ar} / \mathrm{O}-\mathrm{O} / \mathrm{H}$ relation at very high metallicity regime, which is inaccessible in $\mathrm{SF}$ abundance studies.

In this context, we used the $T_{\mathrm{e}}$-method and developed a new methodology based on photoionization model to calculate the abundance of the argon relative to hydrogen in the narrow-line regions (NLRs) of Seyfert 2 galaxies, whose data were taken from the SDSS-DR7 (York et al. 2000). The present study is organized as follows. In Section 2, the observational data and the methodology used to estimate the oxygen and argon abundances are presented. The results and the discussion are presented in Sect. 3. Finally, the conclusion of the outcome is given in Sect. 4 .

1 For a discussion on the universality of the IMF see, for example, Bastian et al. (2010). 




Figure 1. Diagnostic diagram $\log \left(\left[\mathrm{O}_{\mathrm{III}}\right] \lambda 5007 / \mathrm{H} \beta\right)$ versus $\log \left(\left[\mathrm{N}_{\mathrm{II}}\right] \lambda 6584 / \mathrm{H} \alpha\right)$. Red points represent our sample of Seyfert 2 nuclei (see Sect. 2.1) whose observational emission-line ratios were taken from the SDSS-DR7 (York et al. 2000) and measured by the MPA/JHU group. The solid black line represents the AGN/Starforming region separation line proposed by Kewley et al. (2001) and given by the Equation 1

\section{METHODOLOGY}

To determine the total abundance of the argon and oxygen in relation to hydrogen abundance $(\mathrm{Ar} / \mathrm{H}, \mathrm{O} / \mathrm{H})$, firstly, we consider optical emission-line intensities of AGNs type Seyfert 2 from Sloan Digital Sky Survey Data Release 7 (SDSS-DR7, York et al. 2000). These observational data were used to calculate the $\mathrm{Ar} / \mathrm{H}$ and $\mathrm{O} / \mathrm{H}$ abundances using the $T_{\mathrm{e}}$-method. We used photoionization models, built with the Cloudy code (Ferland et al. 2017), in order to obtain an ICF for the $\mathrm{Ar}^{2+}$ and an estimation of the temperature for the gas region occupied by this ion. In what follows, a description of the observational data and the methodology adopted to obtain the abundances are presented.

\subsection{Observational data}

We used optical $(3000<\lambda(\AA)<7200)$ reddening-corrected emission-line intensities of a sample of Seyfert 2 nuclei obtained from the SDSS-DR7 (York et al. 2000) data made available by MPA/JHU group ${ }^{2}$. The sample consists of 463 Seyfert 2 nuclei with redshift $z \lesssim 0.4$ and stellar masses of the hosting galaxies in the range of $9.4 \lesssim \log \left(M / \mathrm{M}_{\odot}\right) \lesssim 11.6$ selected by Dors et al. (2020b). From this sample, we considered a sub-sample containing only objects which have the

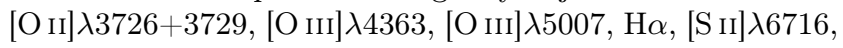
[S II] $\lambda 6731$ and [Ar III] $\lambda 7135$ emission-lines measured with an error lower than $50 \%$. This criterion reduced the sample to 64 objects out of the 463 selected by Dors et al. (2020b), with redshif in the range of $0.04 \lesssim z \lesssim 0.25$ and range of the stellar masses of the hosting galaxies $9.9 \lesssim \log \left(M / \mathrm{M}_{\odot}\right) \lesssim 11.2$.

In Figure 1, a standard Baldwin, Phillips \& Terlevich diagram (Baldwin et al. 1981) $\log ([\mathrm{O}$ III $] \lambda 5007 / \mathrm{H} \beta$ ) versus $\log \left(\left[\mathrm{N}_{\mathrm{II}}\right] \lambda 6584 / \mathrm{H} \alpha\right)$, the observational line ratio intensities

${ }^{2}$ https://wwwmpa.mpa-garching.mpg.de/SDSS/DR7/

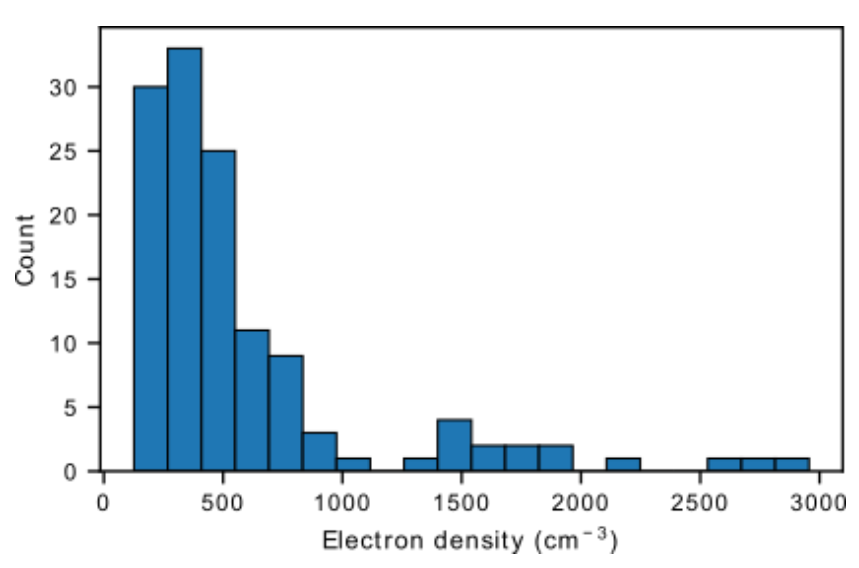

Figure 2. Histogram showing the distribution of electron density values from our sample of 64 objects (see Sect. 2.1) calculated with PyNeb routine (Luridiana et al. 2015) and considering an electron temperature of $10^{4} \mathrm{~K}$. The y-axis represents the number of object with a given range of electron density value.

for the 64 objects are represented by red points. Also in this figure, the theoretical classification criterion (represented by the black line) proposed by Kewley et al. (2001), which depict that objects with

$$
\log ([\mathrm{O} \text { III }] \lambda 5007 / \mathrm{H} \beta)>\frac{0.61}{\log ([\mathrm{N} \text { II }] \lambda 6584 / \mathrm{H} \alpha)-0.47}+1.19
$$

are classified as AGNs, otherwise, as SFs, is shown. It can be seen that the sample cover a large range of ionization degree and metallicity hence a wide range of $[\mathrm{O} \mathrm{III]} / \mathrm{H} \beta$ and $[\mathrm{N}$ II] $/ \mathrm{H} \alpha$ are observed (e.g. Groves et al. 2006; Feltre et al. 2016; Carvalho et al. 2020).

The electron density $\left(N_{\mathrm{e}}\right)$ of each one of the 64 Seyfert 2 nuclei was calculated from the $\left[\mathrm{S}_{\mathrm{II}}\right] \lambda 6716 / \lambda 6731$ line ratio, assuming an electron temperature of $10000 \mathrm{~K}$, and using the PyNeB routine (Luridiana et al. 2015). In Fig. 2 a histogram with the $N_{\mathrm{e}}$ distribution of our sample is shown. It can be seen that the $N_{\mathrm{e}}$ values for our sample (the maximum value is about $3000 \mathrm{~cm}^{-3}$ ) are lower than the critical density (i.e. $10^{4-8} \mathrm{~cm}^{-3}$, see Vaona et al. 2012) of the emission lines involved in the present study, therefore, effects of collisional de-excitation are negligible in our abundance estimates. In Dors et al. (2020b), a complete description of the selection criteria adopted to obtain the sample as well as a discussion about aperture effects on the abundance determination is presented. Moreover, effects of electron density variation along the AGN radius, X-Ray dominated regions, shock and electron temperature fluctuations in abundance determinations have been discussed by Dors et al. (2020a) and Dors et al. (2021) and these are not repeated here.

\section{$2.2 \quad T_{\mathrm{e}}$-method}

It was possible to estimate the $\mathrm{Ar} / \mathrm{H}$ and $\mathrm{O} / \mathrm{H}$ abundances through the $T_{\mathrm{e}}$-method for the sample of 64 objects. In view of the $T_{\mathrm{e}}$-method, we followed a similar methodology proposed by Pérez-Montero (2017) and Dors et al. (2020a). 


\subsubsection{Oxygen abundance}

First, for each object, we calculated the temperature of the high ionisation gas zone $\left(t_{3}\right)$ and the electron density $\left(N_{\mathrm{e}}\right)$ based on the dependence of these nebular parameters on the $[\mathrm{O}$ III $](\lambda 4949+\lambda 5007) / \lambda 4363$ and $[\mathrm{S}$ II $] \lambda 6716 / \lambda 6731$ line ratios, respectively. We used the function GETCRossTEMDen from the PyNeB code (Luridiana et al. 2015), where the value of each parameter was obtained by interacting over the two sensitive line ratios above. The errors in $N_{\mathrm{e}}$ and $t_{3}$ were calculated adding a Monte Carlo random-gauss values to the sample with the function ADDMONTECARLOOBS in the PyNeB code. The symbol $t_{3}$ represents the electron temperature in units of $10^{4} \mathrm{~K}$. The mean value derived for $t_{3}$ from our sample is $\sim 1.5$ and the uncertainty is $\sim 0.3$. For the electron density, we found a mean value of $470 \mathrm{~cm}^{-3}$ and an uncertainty of $\sim 250 \mathrm{~cm}^{-3}$.

Since it is not possible to estimate the temperature for the low ionization gas zone $\left(t_{2}\right)$ due to the absence of the auroral $\left[\mathrm{N}_{\mathrm{II}}\right] \lambda 5755$ and $[\mathrm{O} \mathrm{II}] \lambda 7319, \lambda 7330$ line intensities, the following theoretical relation (Eq. 2) between $t_{2}-t_{3}$, derived by Dors et al. (2020a) for chemical abundance studies of AGNs and obtained by using a photoionzation model grid built with the CLOudy code (Ferland et al. 2013) by Carvalho et al. (2020), was adopted:

$t_{2}=\left(\mathrm{a} \times t_{3}^{3}\right)+\left(\mathrm{b} \times t_{3}^{2}\right)+\left(\mathrm{c} \times t_{3}\right)+\mathrm{d}$,

where $\mathrm{a}=0.17, \mathrm{~b}=-1.07, \mathrm{c}=2.07$ and $\mathrm{d}=-0.33$, while $t_{2}$ and $t_{3}$ are in units of $10^{4} \mathrm{~K}$. In Riffel et al. (2021) this theoretical $t_{2}-t_{3}$ relation was compared with direct electron temperature estimations, calculated through observational auroral emission lines for a sample of AGNs, and a good agreement was found between them. However, these authors indicated that some cautions must be taken into account in the use of Eq. 2 for AGNs with strong outflowing gas.

To calculate the ionic abundances of $\mathrm{O}^{2+}$ and $\mathrm{O}^{+}$relative to $\mathrm{H}^{+}$the expressions provided by Pérez-Montero (2017):

$$
\begin{array}{r}
12+\log \left(\frac{\mathrm{O}^{2+}}{\mathrm{H}^{+}}\right)=\log \left(\frac{1.33 \times I([\mathrm{O} \mathrm{III}] \lambda 5007)}{I(\mathrm{H} \beta)}\right)+6.1868 \\
+\frac{1.2491}{t_{3}}-0.55816 \log \left(t_{3}\right)
\end{array}
$$

and

$$
\begin{aligned}
12+\log \left(\frac{\mathrm{O}^{+}}{\mathrm{H}^{+}}\right) & =\log \left(\frac{I([\mathrm{O} \mathrm{II}] \lambda 3726+\lambda 3729)}{I(\mathrm{H} \beta)}\right)+5.887 \\
& +\frac{1.641}{t_{2}}-0.543 \log \left(t_{2}\right)+0.000114 n_{\mathrm{e}}
\end{aligned}
$$

respectively, where $n_{\mathrm{e}}$ is the electron density $N_{\mathrm{e}}$ in units of $10^{4} \mathrm{~cm}^{-3}$, were used. The uncertainty of $t_{3}$ was considered in the determination of $t_{2}$. Thus, in the derivation of $\mathrm{O}^{2+} / \mathrm{H}^{+}$ ionic abundance the uncertainties of the $[\mathrm{O} \mathrm{III}] / \mathrm{H} \beta$ line ratio and of $t_{3}$ were taken into account. The same procedure was considered for the derivation of $\mathrm{O}^{+} / \mathrm{H}^{+}$, where the $n_{\mathrm{e}}$ and $t_{2}$ uncertainties were also taken into account.

Finally, the total abundance of the oxygen is approximated by

$\frac{\mathrm{O}}{\mathrm{H}}=\mathrm{ICF}(\mathrm{O}) \times\left[\frac{\mathrm{O}^{2+}}{\mathrm{H}^{+}}+\frac{\mathrm{O}^{+}}{\mathrm{H}^{+}}\right]$.

It is not possible to derive $\operatorname{ICF}(\mathrm{O})$, for instance through the expressions proposed by Torres-Peimbert \& Peimbert (1977) and Izotov et al. (2006), due to the absence of the He II $\lambda 4686$ emission line in our observational data. Therefore, we assumed $\operatorname{ICF}(\mathrm{O})=1.20$, an average value obtained by Flury \& Moran (2020) and Dors et al. (2020a). Oxygen ICF values translates into an abundance correction for AGNs of $\sim 0.1$ dex, which is in agreement with the uncertainty derived in $\mathrm{H}$ II regions via $T_{\mathrm{e}}$-method estimates (e.g. Kennicutt et al. 2003; Hägele et al. 2008; Berg et al. 2020).

\subsubsection{Argon abundance}

Generally, to estimate the temperature of the electrons excitating the $\mathrm{Ar}^{2+}$, the approach proposed by Garnett (1992) which is based on photoionization model results is assumed in chemical abundance studies of SFs (e.g. Kennicutt et al. 2003; Hägele et al. 2008). A similar electron temperature is assumed for the gas regions where the $\mathrm{Ar}^{2+}$ and $\mathrm{S}^{2+}$ ions are located, i.e.

$T_{\mathrm{e}}(\mathrm{Ar}$ III $)=T_{\mathrm{e}}(\mathrm{S}$ III $)$.

The $T_{\mathrm{e}}(\mathrm{S}$ III $)$ can be calculated directly from $[\mathrm{S}$ III $](\lambda 9069+\lambda 9532) / \lambda 6312$ or through the following theoretical relation proposed by Garnett (1992), i.e.

$T_{\mathrm{e}}(\mathrm{S}$ III $)=0.83 T_{\mathrm{e}}(\mathrm{O}$ III $)+1700 \mathrm{~K}$

when $[\mathrm{S}$ III $](\lambda 9069+\lambda 9532) / \lambda 6312$ line ratio can not be measured. In Eq. $7, T_{\mathrm{e}}(\mathrm{O}$ III $)=t_{3} \times 10^{4} \mathrm{~K}$.

However, Dors et al. (2020a) showed that, in general, AGNs present a distinct electron temperature structure than H II regions (see also Dors et al. 2021; Riffel et al. 2021; Armah et al. 2021). Thus, it is worthwhile to ascertain the validity of Eqs. 6 and 7 for AGNs. In view of this, we used the results from the photoionization model grid by Carvalho et al. (2020) and considered predictions for $T_{\mathrm{e}}$ (A III) and $T_{\mathrm{e}}$ (S III). This grid of photoionization models considered a wide range of AGN nebular parameters whose optical predicted emission lines reproduce those of a large sample of Seyfert 2 nuclei (for a detailed description of these models see Carvalho et al. 2020). The temperature values predicted by the photoionization models and considered here correspond to the mean temperature for $\mathrm{Ar}^{2+}$ and $\mathrm{S}^{2+}$ over the nebular AGN radius times the electron density. In Fig. 3, bottom panel, the photoionization model predictions for $T_{\mathrm{e}}\left(\mathrm{S}\right.$ III) versus $T_{\mathrm{e}}\left(\mathrm{Ar}\right.$ III) (in units of $10^{4} \mathrm{~K}$ ) and the approach given by Eq. 6 are shown. It can be seen that, in contrast to $\mathrm{H}$ II regions, $T_{\mathrm{e}}$ (A III) is generally higher than $T_{\mathrm{e}}$ (S III), indicating that Eq. 6 is not valid for AGN abundance studies. The same can be seen in Fig. 3, top panel, where the AGN model predictions show a large deviation from the temperature relation given by Eq. 7 .

In order to estimate a more precise temperature to derive the $\mathrm{Ar}^{2+} / \mathrm{H}^{+}$ionic abundance, the photoionization models by Carvalho et al. (2020) were used to obtain a relation between $T_{\mathrm{e}}\left(\mathrm{Ar}\right.$ III) and $t_{3}$ as shown in Fig. 4 because $t_{3}$ can be derived directly from $[\mathrm{O}$ III $](\lambda 4959+\lambda 5007) / \lambda 4363$ observational line ratio of our sample. In Fig. $4, t_{\mathrm{e}}(\mathrm{Ar}$ III $)$ corresponds to the value of $T_{\mathrm{e}}(\mathrm{Ar}$ III $)$ in units of $10^{4} \mathrm{~K}$. It can be seen from Fig. 4 that there is a good correlation between the temperatures and a fit to the points results in 

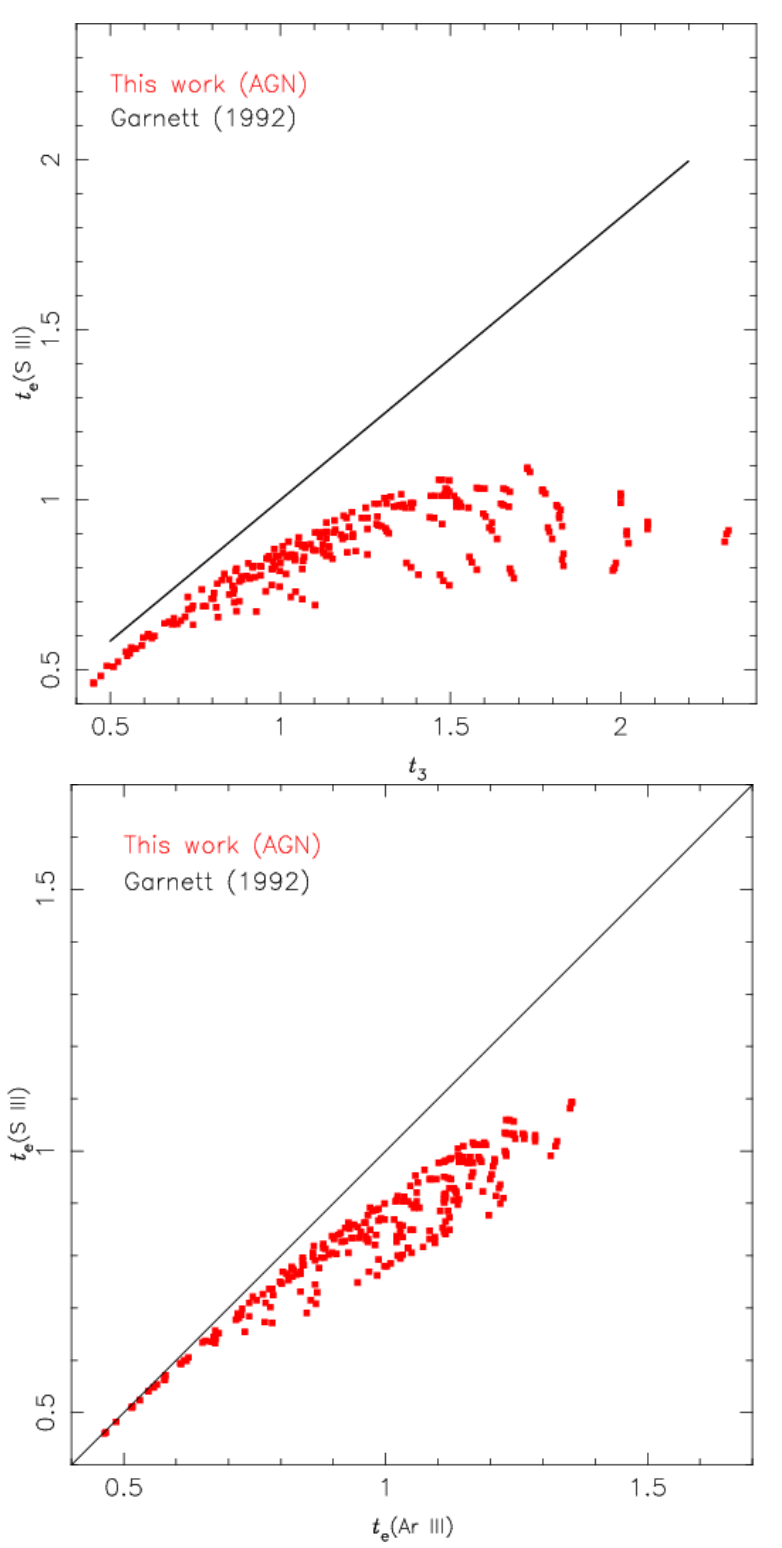

Figure 3. Temperature values for the $\mathrm{S}^{2+}, \mathrm{Ar}^{2+}$ and $\mathrm{O}^{2+}$ predicted by the photoionization models built by Carvalho et al. (2020) by using the Cloudy code (Ferland et al. 2013). The values correspond to the model predicted mean temperature (in units of $10^{4} \mathrm{~K}$ ) for each ion over the nebular AGN radius times the electron density. Bottom panel: temperature values for the $\mathrm{S}^{2+}$ versus those for $\mathrm{A}^{2+}$. The black line corresponds to equality between the temperatures (Eq. 6) proposed by Garnett (1992) for $\mathrm{H}$ II regions. Red points represent photoionization model results. Top panel: same as the bottom panel but for $\mathrm{S}^{2+}$ versus $t_{3}$, where $t_{3}$ represents the temperature for $\mathrm{O}^{2+}$. Black line represents Eq. 7 proposed by Garnett (1992).

The $\mathrm{Ar}^{2+} / \mathrm{H}^{+}$ionic abundance is calculated by the expression also provided by Pérez-Montero (2017)

$$
\begin{aligned}
12+\log \left(\frac{\mathrm{Ar}^{2+}}{\mathrm{H}^{+}}\right)= & \log \left(\frac{I[\mathrm{Ar} I \mathrm{II}] \lambda 7135}{I(\mathrm{H} \beta)}\right)+6.100 \\
& +\frac{0.86}{t_{\mathrm{e}}(\operatorname{Ar} \mathrm{III})}-0.404 \times \log t_{\mathrm{e}}(\text { Ar III }) .(9)
\end{aligned}
$$

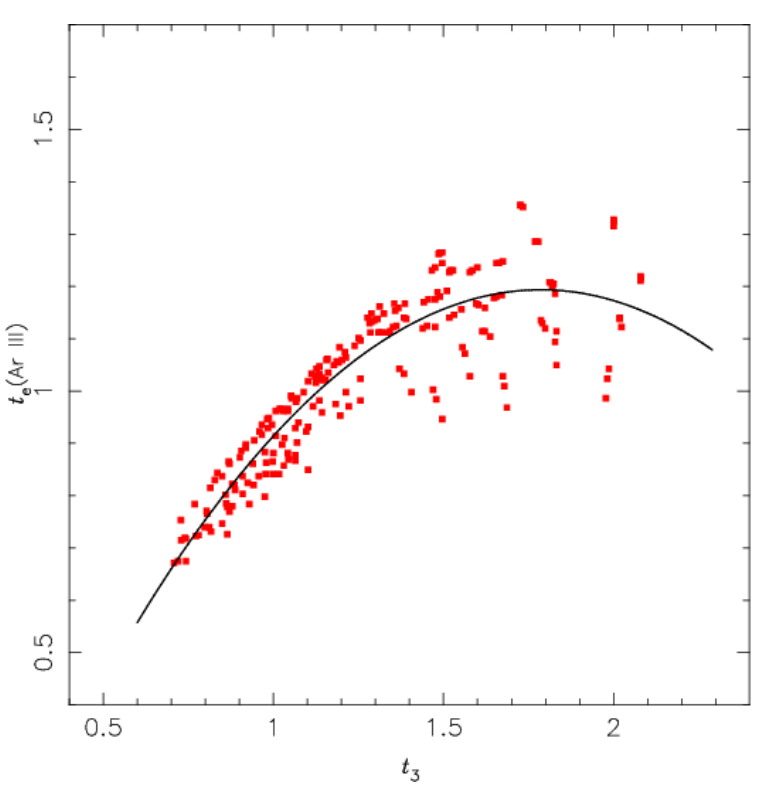

Figure 4. Same as Fig. 3 but for $T_{\mathrm{e}}\left(\mathrm{Ar}\right.$ III) versus $t_{3}$. Black curve represents a fit to the points represented by Eq. 8 .

To calculate the total argon abundance in relation to the hydrogen $(\mathrm{Ar} / \mathrm{H})$ using only the $\mathrm{Ar}^{2+} / \mathrm{H}^{+}$abundance it is necessary to consider an ICF in order to take into account the presence of ions with other ionization levels. In fact, ions such as $\mathrm{Ar}^{3+}$ and $\mathrm{Ar}^{4+}$ have emission lines (e.g. [Ar IV] $\lambda 4740,[$ Arv] $\lambda 7006)$ present in optical AGN spectra (e.g. Koski 1978; Alloin et al. 1992). Hitherto, no ICF for the argon abundance determinations in AGNs has been proposed in the literature and, in principle, due to distinct ionization structure of AGNs, theoretical ICFs proposed by H II regions (e.g. Mathis 1985; Izotov et al. 2006; Pérez-Montero et al. 2007; Berg et al. 2020; Amayo et al. 2021) can not be applied to this class of objects. In this sense, we used the Carvalho et al. (2020) photoionization models to derive an argon ICF, defined by

$\operatorname{ICF}\left(\operatorname{Ar}^{2+}\right)=(\operatorname{Ar} / \mathrm{H}) /\left(\mathrm{Ar}^{2+} / \mathrm{H}^{+}\right)$

and in terms of the function $\mathrm{x}=\left[\mathrm{O}^{2+} /\left(\mathrm{O}^{+}+\mathrm{O}^{2+}\right)\right]$ ionic abundance ratio. In Fig. 5 the results for $\operatorname{ICF}\left(\mathrm{Ar}^{2+}\right)$ versus $\mathrm{x}$ are shown. A fit to the points produces the relation

$\operatorname{ICF}\left(\mathrm{Ar}^{+}\right)=\left(\mathrm{ax}^{2}\right)+(\mathrm{bx})+\mathrm{c}$

where $\mathrm{a}=20.88 \pm 3.80, \mathrm{~b}=-9.74 \pm 3.64$ and $\mathrm{c}=2.68 \pm$ 0.69. A large scattering of the points can be seen in Fig. 5 . We investigated the source of this scattering to ascertain whether it is due to variation in the nebular parameters $N_{\mathrm{e}}, \alpha_{o x}$ (slope of the Spectral Energy Distribution, $\mathrm{SED}^{3}$ ) and ionization parameter $U$ (not shown). We did not find any dependence of these parameters with the $\operatorname{ICF}\left(\mathrm{Ar}^{2+}\right)-\mathrm{x}$ relation. However, a clear dependence between $\operatorname{ICF}\left(\mathrm{Ar}^{2+}\right)$ and $\mathrm{x}$ on the gas metallicity is noted in Fig. 5, in the sense that for a fixed $\mathrm{x}$ value, we derive a higher ICF value with the decrease of the metallicity. A dependence between the metallicity and ICF-x relations for distinct elements was also derived by Izotov et al. (2006) for SFs.

${ }^{3}$ See Krabbe et al. 2021 for a detailed description of this SED. 




Figure 5. ICF for $\mathrm{Ar}^{2+}$ (as defined in Eq. 10) as a function of the $\mathrm{x}=\left[\mathrm{O}^{2+} /\left(\mathrm{O}^{+}+\mathrm{O}^{2+}\right)\right]$ abundance ratio. Points represent results from photoionization models built by Carvalho et al. (2020). Model results assuming different metallicities (in relation to the solar value) are represented by different colours, as indicated. The curve represents a fit to all the points given by the Eq. 11 .

Thus, in order to obtain a more exact ICF derivation, we produce a bi-parametric function, i.e. $\operatorname{ICF}\left(\mathrm{Ar}^{2+}\right)=\mathrm{f}[12+$ $\log (\mathrm{O} / \mathrm{H}), \mathrm{x}]$. We converted the metallicity $(Z)$ assumed in the photoionization models built by Carvalho et al. (2020) in oxygen abundance through the expression

$12+\log (\mathrm{O} / \mathrm{H})=12+\log \left[\left(Z / \mathrm{Z}_{\odot}\right) \times 10^{\log (\mathrm{O} / \mathrm{H}) \odot],}\right.$

where $\log (\mathrm{O} / \mathrm{H})_{\odot}=-3.31$ (Allende Prieto et al. 2001). In Fig. 6 the bi-parametric calibration is shown where the points represent the photoionization model results. We fit two functions, one for $\mathrm{x}<0.6$ represented by a blue surface in Fig. 6 and given by

$$
\begin{aligned}
\operatorname{ICF}\left(\mathrm{Ar}^{2+}\right) & =(1.51 \pm 0.3) x^{2}-(6.82 \pm 1.55) y^{2} \\
& -(1.68 \pm 0.52) x y-(27.18 \pm 5.19) x \\
& +(10.88 \pm 4.72) y+124.6 \pm 22.32
\end{aligned}
$$

where $\mathrm{y}=12+\log (\mathrm{O} / \mathrm{H})$ and $\mathrm{x}=\left[\mathrm{O}^{2+} /\left(\mathrm{O}^{+}+\mathrm{O}^{2+}\right)\right]$ and another one for $\mathrm{x}>0.6$ represented by the green surface and given by

$$
\begin{aligned}
\mathrm{ICF}\left(\mathrm{Ar}^{2+}\right)= & (1.82 \pm 142.29) x^{2}+(107.86 \pm 21.07) y^{2} \\
- & (35.61 \pm 7.13) x y-(14.54 \pm 33.81) x \\
& +(188 \pm 53.96) y+27.73 \pm 142.29
\end{aligned}
$$

The expressions above were applied to derive the total abundance of the argon in relation to the hydrogen $(\mathrm{Ar} / \mathrm{H})$.

The mean errors for the $12+\log (\mathrm{Ar} / \mathrm{H})$ and $12+\log (\mathrm{O} / \mathrm{H})$ abundances derived for the objects of our sample are $\sim 0.25 \mathrm{dex}$ and $\sim 0.13 \mathrm{dex}$, respectively. These errors are in order of those derived for $\mathrm{O} / \mathrm{H}$ abundance determinations in nearby AGNs by Revalski et al. (2018a,b, 2021), who also applied the $T_{\mathrm{e}}$-method. However, they are somewhat $(\sim 0.1$ dex $)$ higher than those derived for disk $\mathrm{H}_{\mathrm{II}}$ regions located in nearby galaxies (e.g. Berg et al.

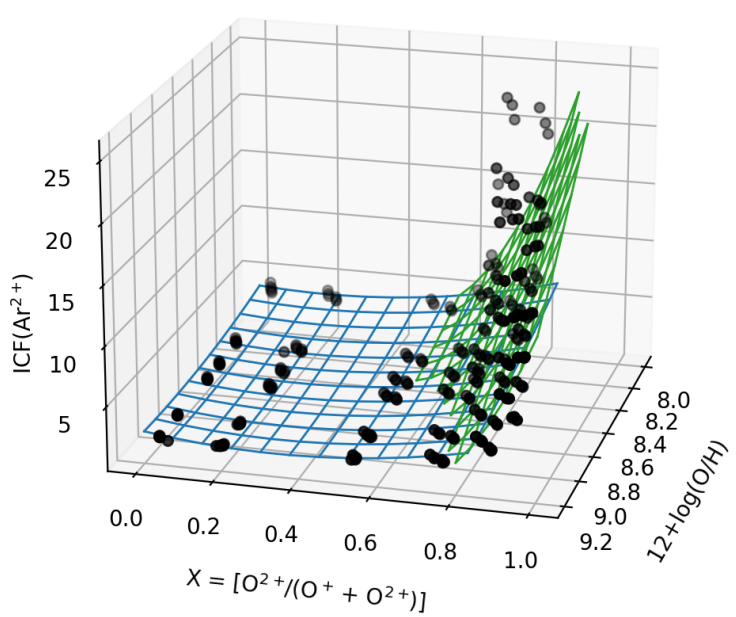

Figure 6. Bi-parametric calibration among the $\mathrm{ICF}\left(\mathrm{Ar}^{2+}\right), 12+$ $\log (\mathrm{O} / \mathrm{H})$ and $\mathrm{x}=\left[\mathrm{O}^{2+} /\left(\mathrm{O}^{+}+\mathrm{O}^{2+}\right)\right]$. Points represent the photoionization model results built by Carvalho et al. (2020) while the surfaces represent the best fit to the points. The blue surface is given by Eq. 13 and valid it is for $\mathrm{x}<0.6$ while the green surface, given by Eq. 14, is valid for $\mathrm{x}>0.6$.

2020), because the $[\mathrm{O} \mathrm{II}] \lambda 4363$ auroral line is weaker in AGNs than in $\mathrm{H}$ II regions, resulting in a higher uncertainty in its flux, which in turn implies a larger uncertainty in the AGN abundance values.

In Table 1, the SDSS identification of the objects of the sample, electron temperature and electron density values, argon ICF values, ionic and total abundances, redshift, and stellar mass of the hosting galaxies are listed.

\section{RESULTS AND DISCUSSION}

The total argon abundance relative to hydrogen $(\mathrm{Ar} / \mathrm{H})$ in gaseous nebulae has been derived mainly through measurements of the $[\mathrm{Ar}$ III] $\lambda 7135 / \mathrm{H} \beta$ and the assumptions considered to convert this line ratio into the $\mathrm{Ar}^{2+} / \mathrm{H}^{+}$ionic abundance as well as by using an expression for the $\operatorname{ICF}\left(\mathrm{Ar}^{2+}\right)$ (e.g. Kennicutt et al. 2003). In some few cases, it has been also possible to measure the $[\mathrm{Ar} \mathrm{IV}] \lambda 4740$ and $[\mathrm{Ar} \mathrm{v}] \lambda 7006$ emission lines and deriving the $\mathrm{Ar}^{3+} / \mathrm{H}^{+}$and $\mathrm{Ar}^{4+} / \mathrm{H}^{+}$ ionic abundances, respectively (e.g. Koski 1978; French 1981; Pagomenos et al. 2018), producing a more exact $\mathrm{Ar} / \mathrm{H}$ determination, i.e.

$\frac{\mathrm{Ar}}{\mathrm{H}}=\mathrm{ICF}(\mathrm{Ar}) \times\left[\frac{\mathrm{Ar}^{2+}}{\mathrm{H}^{+}}+\frac{\mathrm{Ar}^{3+}}{\mathrm{H}^{+}}+\frac{\mathrm{Ar}^{4+}}{\mathrm{H}^{+}}\right]$

(e.g. Wesson et al. 2005). However, the [Ar IV] $\lambda 4740$ and $[$ Ar v] $\lambda 7006$ emission lines are weak in most part of low ionization objects, therefore, it is most convenient to use only the strong [Ar III] $\lambda 7135$ (e.g. Kennicutt et al. 2003; Zurita \& Bresolin 2012). In our case, the emission line measurements made available by the MPA/JHU group only allowed us to derive $\mathrm{Ar}^{2+} / \mathrm{H}^{+}$ionic abundance and then apply the ICF (Eq. 13) to obtain the total argon abundance (Ar/H). The application of the $T_{\mathrm{e}}$-method to AGNs was recently 
Table 1. Results obtained from our sample of objects. Columns: (1) SDSS name, (2) $T_{\mathrm{e}}\left(\mathrm{O}\right.$ IIII) (in K), (3) $T_{\mathrm{e}}(\mathrm{A}$ IıI) (in K), (4) Electron density (in units of $\left.\mathrm{cm}^{-3}\right),(5) \mathrm{ICF}\left(\mathrm{Ar}^{2+}\right),(6) 12+\log \left(\mathrm{O}^{+} / \mathrm{H}^{+}\right),(7) 12+\log \left(\mathrm{O}^{2+} / \mathrm{H}^{+}\right),(8) 12+\log \left(\mathrm{A}^{2+} / \mathrm{H}^{+}\right),(9) 12+\log (\mathrm{O} / \mathrm{H}),(10)$ $12+\log (\mathrm{Ar} / \mathrm{H}),(11)$ redshift and $(12)$ Mass (in units of $10^{7} \mathrm{M}_{\odot}$ ).

\begin{tabular}{|c|c|c|c|c|c|c|c|c|c|c|c|}
\hline (1) & 6) & $(3)$ & (4) & $(5)$ & $(6)$ & $(7)$ & $(8)$ & $(9)$ & $(10)$ & $(11)$ & $(12)$ \\
\hline J101754.72-002811.9 & 14510 & 11481 & 298 & 2.79 & 8.03 & 7.87 & 5.98 & 8.34 & 6.43 & 0.1817 & 11.13 \\
\hline $\mathrm{J} 101536.21+005459.3$ & 18252 & 11994 & 184 & 2.14 & 8.39 & 7.78 & 5.60 & 8.56 & 5.93 & 0.1202 & 10.71 \\
\hline $\mathrm{J} 104426.16+001707.3$ & 8872 & 8343 & 292 & 2.40 & 8.30 & 8.41 & 6.31 & 8.74 & 6.69 & 0.1502 & 10.71 \\
\hline J105408.69-000111.0 & 16992 & 11971 & 227 & 2.39 & 8.42 & 7.33 & 5.36 & 8.53 & 5.74 & 0.1081 & 10.21 \\
\hline J111652.97+010615.5 & 16865 & 11952 & 259 & 2.84 & 8.05 & 7.49 & 5.54 & 8.23 & 6.00 & 0.1309 & 10.70 \\
\hline J114017.31-001543.3 & 13459 & 10677 & 505 & 2.46 & 8.17 & 7.99 & 6.09 & 8.47 & 6.48 & 0.1245 & 10.62 \\
\hline $\mathrm{J} 113049.84+005346.7$ & 19873 & 11827 & 600 & 2.35 & 8.37 & 7.49 & 5.62 & 8.50 & 5.99 & 0.1043 & 10.70 \\
\hline $\mathrm{J} 113326.76+001443.9$ & 14509 & 11386 & 497 & 2.61 & 8.16 & 7.54 & 5.62 & 8.33 & 6.03 & 0.1143 & 10.48 \\
\hline J115616.76-002221.0 & 15385 & 11724 & 781 & 2.16 & 8.31 & 7.96 & 6.40 & 8.55 & 6.74 & 0.1092 & 10.74 \\
\hline J122012.58-010531.5 & 11277 & 10039 & 1443 & 2.18 & 8.39 & 8.43 & 6.24 & 8.79 & 6.58 & 0.1183 & 10.55 \\
\hline J123441.93-010034.7 & 14568 & 11518 & 381 & 2.52 & 8.15 & 7.75 & 5.55 & 8.37 & 5.95 & 0.0801 & 10.25 \\
\hline $\mathrm{J} 124116.14+004423.0$ & 14438 & 11537 & 659 & 2.24 & 8.26 & 8.02 & 5.90 & 8.54 & 6.26 & 0.0900 & 10.74 \\
\hline $\mathrm{J} 130433.90+000402.9$ & 16195 & 11896 & 158 & 2.56 & 8.19 & 7.53 & 5.92 & 8.35 & 6.33 & 0.2463 & 11.23 \\
\hline J132625.73-002148.6 & 17204 & 11988 & 273 & 2.89 & 8.10 & 7.25 & 5.53 & 8.24 & 5.99 & 0.1893 & 10.78 \\
\hline J134005.97-010646.4 & 20688 & 11681 & 189 & 2.35 & 8.48 & 7.29 & 5.43 & 8.59 & 5.81 & 0.1295 & 10.77 \\
\hline $\mathrm{J} 133821.79+002329.2$ & 12623 & 10797 & 233 & 2.60 & 8.17 & 8.25 & 6.14 & 8.59 & 6.55 & 0.1292 & 10.87 \\
\hline $\mathrm{J} 140301.05+005343.5$ & 14002 & 11329 & 155 & 3.03 & 7.94 & 7.65 & 5.51 & 8.20 & 5.99 & 0.1664 & 10.61 \\
\hline J145956.36-002821.5 & 12503 & 10682 & 202 & 2.57 & 8.13 & 8.01 & 6.14 & 8.45 & 6.55 & 0.1096 & 10.84 \\
\hline J130354.71-030631.8 & 11344 & 10073 & 757 & 1.84 & 8.53 & 8.40 & 6.28 & 8.85 & 6.55 & 0.0778 & 10.65 \\
\hline $\mathrm{J} 171544.02+600835.4$ & 17771 & 12000 & 819 & 2.14 & 8.38 & 7.79 & 5.59 & 8.56 & 5.92 & 0.1569 & 10.98 \\
\hline $\mathrm{J} 172028.98+584749.6$ & 14587 & 11494 & 494 & 2.92 & 8.03 & 7.42 & 5.29 & 8.21 & 5.75 & 0.1269 & 10.84 \\
\hline $\mathrm{J} 172352.43+582318.5$ & 17778 & 11997 & 939 & 2.91 & 8.07 & 7.31 & 5.20 & 8.22 & 5.66 & 0.0799 & 10.24 \\
\hline $\mathrm{J} 153035.77+001517.7$ & 11505 & 10165 & 207 & 2.28 & 8.24 & 7.93 & 5.87 & 8.50 & 6.23 & 0.0721 & 10.53 \\
\hline J002312.34+003956.3 & 19764 & 11869 & 447 & 2.06 & 8.54 & 7.73 & 5.67 & 8.68 & 5.98 & 0.0727 & 10.16 \\
\hline J012937.25-003838.6 & 14176 & 11401 & 141 & 2.57 & 8.16 & 7.60 & 5.51 & 8.34 & 5.92 & 0.1794 & 11.06 \\
\hline J012720.32+010214.6 & 9838 & 9066 & 498 & 2.27 & 8.41 & 8.55 & 6.36 & 8.87 & 6.72 & 0.1745 & 11.09 \\
\hline J013957.81-004504.2 & 11872 & 10381 & 441 & 2.74 & 8.11 & 8.18 & 6.40 & 8.52 & 6.83 & 0.1616 & 10.83 \\
\hline J014153.97+010505.4 & 19553 & 11866 & 581 & 2.23 & 8.40 & 7.62 & 5.33 & $8.5 \overline{5}$ & 5.68 & 0.1013 & 10.95 \\
\hline J011016.00+150515.9 & 12920 & 10890 & 534 & 2.28 & 8.27 & 8.19 & 6.10 & 8.61 & 6.46 & 0.0597 & 10.19 \\
\hline J013555.82+143529.6 & 12902 & 10883 & 267 & 2.52 & 8.14 & 7.86 & 5.68 & 8.40 & 6.08 & 0.0719 & 10.83 \\
\hline J074213.71 + 391705.3 & 10451 & 9509 & 201 & 2.40 & 8.22 & 8.19 & 6.19 & 8.59 & 6.57 & 0.0704 & 9.94 \\
\hline J082017.99+465125.3 & 12773 & 10823 & 130 & 2.13 & 8.46 & 7.70 & 5.64 & 8.61 & 5.97 & 0.0524 & 10.35 \\
\hline J082910.18+504005.7 & 11671 & 10220 & 205 & 2.00 & 8.44 & 7.92 & 5.91 & 8.64 & 6.21 & 0.0739 & 9.99 \\
\hline J085223.96+531550.6 & 17610 & 11995 & 524 & 2.52 & 8.27 & 7.40 & 5.54 & 8.41 & 5.94 & 0.1280 & 10.78 \\
\hline J095123.44+581621.2 & 18905 & 11952 & 140 & 2.45 & 8.48 & 6.96 & 5.12 & 8.58 & 5.51 & 0.1486 & 10.91 \\
\hline J033923.14-054841.5 & 12841 & 10856 & 331 & 2.54 & 8.16 & 8.13 & 5.86 & 8.52 & 6.27 & 0.0848 & 10.26 \\
\hline J091605.16+002030.3 & 12682 & 10791 & 482 & 1.83 & 8.53 & 8.14 & 5.77 & 8.76 & 6.03 & 0.1434 & 10.97 \\
\hline J093509.12+002557.4 & 15921 & 11833 & 551 & 2.70 & 8.11 & 7.52 & 5.24 & 8.29 & 5.68 & 0.1512 & 10.78 \\
\hline $\mathrm{J} 100013.84+624703.4$ & 10996 & 9868 & 488 & 2.55 & 8.15 & 8.09 & 5.96 & 8.50 & 6.37 & 0.1145 & 10.33 \\
\hline $\mathrm{J} 102039.81+642435.8$ & 15695 & 11779 & 172 & 2.29 & 8.25 & 7.86 & 5.47 & 8.47 & 5.83 & 0.1223 & 10.74 \\
\hline J095759.45+022810.5 & 12988 & 10905 & 325 & 2.35 & 8.23 & 8.11 & 6.12 & 8.55 & 6.49 & 0.1194 & 10.56 \\
\hline $\mathrm{J} 100921.26+013334.5$ & 13197 & 11029 & 735 & 2.20 & 8.32 & 8.27 & 6.04 & 8.68 & 6.39 & 0.1437 & 10.72 \\
\hline $\mathrm{J} 112748.89+020302.6$ & 16053 & 11845 & 451 & 2.25 & 8.30 & 7.76 & 5.53 & 8.49 & 5.88 & 0.1267 & 10.80 \\
\hline $\mathrm{J} 114304.62+013946.2$ & 10858 & 9810 & 197 & 2.03 & 8.38 & 8.04 & 5.94 & 8.62 & 6.25 & 0.0928 & 10.84 \\
\hline $\mathrm{J} 114029.55+022744.6$ & 20626 & 11680 & 375 & 2.48 & 8.28 & 7.44 & 5.23 & 8.42 & 5.62 & 0.1230 & 10.63 \\
\hline $\mathrm{J} 115854.96+033254.9$ & 15449 & 11741 & 373 & 2.27 & 8.36 & 7.63 & 5.41 & 8.51 & 5.77 & 0.0841 & 10.51 \\
\hline $\mathrm{J} 125503.63+012233.7$ & 13282 & 11026 & 731 & 2.09 & 8.37 & 8.25 & 5.94 & 8.69 & 6.26 & 0.1642 & 10.93 \\
\hline $\mathrm{J} 125209.68+021558.0$ & 15928 & 11815 & 200 & 2.53 & 8.28 & 7.38 & 5.26 & 8.41 & 5.66 & 0.2064 & 10.95 \\
\hline $\mathrm{J} 130220.35+024048.8$ & 13312 & 11045 & 710 & 2.33 & 8.23 & 7.85 & 5.98 & 8.46 & 6.35 & 0.1766 & 10.93 \\
\hline $\mathrm{J} 134959.37+030058.0$ & 14538 & 11545 & 346 & 2.25 & 8.26 & 7.97 & 6.17 & 8.52 & 6.52 & 0.1097 & 10.35 \\
\hline $\mathrm{J} 140231.58+021546.3$ & 11109 & 9934 & 263 & 2.03 & 8.44 & 8.42 & 6.07 & 8.81 & 6.38 & 0.1797 & 11.06 \\
\hline $\mathrm{J} 143214.54+023228.5$ & 15605 & 11787 & 439 & 2.31 & 8.26 & 7.77 & 5.58 & 8.46 & 5.95 & 0.1123 & 10.91 \\
\hline J074257.23 + 333217.9 & 14084 & 11333 & 366 & 2.77 & 8.05 & 7.66 & 5.53 & 8.27 & 5.97 & 0.1474 & 10.71 \\
\hline J090246.69+520932.8 & 13797 & 11254 & 290 & 2.34 & 8.22 & 8.02 & 5.70 & 8.51 & 6.07 & 0.1375 & 10.95 \\
\hline $\mathrm{J} 141530.97+035916.6$ & 16651 & 11928 & 463 & 2.41 & 8.40 & 7.31 & 5.56 & 8.52 & 5.95 & 0.0805 & 10.49 \\
\hline $\mathrm{J} 141351.75+042208.9$ & 16889 & 11957 & 293 & 2.47 & 8.23 & 7.57 & 5.41 & 8.40 & 5.80 & 0.1449 & 10.60 \\
\hline $\mathrm{J} 144925.29+044157.2$ & 9467 & 8810 & 126 & 1.91 & 8.46 & 8.15 & 6.14 & 8.71 & 6.42 & 0.0824 & 10.04 \\
\hline $\mathrm{J} 151244.15+042848.3$ & 14481 & 11439 & 575 & 2.37 & 8.23 & 7.73 & 5.66 & 8.43 & 6.04 & 0.0796 & 10.34 \\
\hline $\mathrm{J} 155404.39+545708.2$ & 12320 & 10609 & 147 & 2.48 & 8.20 & 8.20 & 6.00 & 8.58 & 6.39 & 0.0457 & 9.91 \\
\hline $\mathrm{J} 164938.71+420658.4$ & 14941 & 11624 & 345 & 2.34 & 8.30 & 7.63 & 5.61 & 8.46 & 5.98 & 0.1503 & 10.58 \\
\hline $\mathrm{J} 165944.29+392846.1$ & 16801 & 11947 & 285 & 2.32 & 8.32 & 7.62 & 5.78 & 8.48 & 6.15 & 0.0818 & 10.09 \\
\hline $\mathrm{J} 100602.50+071131.8$ & 14649 & 11535 & 1588 & 1.87 & 8.64 & 7.95 & 5.76 & 8.80 & 6.04 & 0.1205 & 11.19 \\
\hline $\mathrm{J} 163344.99+372335.1$ & 20228 & 11735 & 771 & 2.50 & 8.21 & 7.57 & 5.54 & 8.38 & 5.94 & 0.1748 & 10.80 \\
\hline $\mathrm{J} 125558.75+291459.4$ & 11324 & 10055 & 366 & 2.00 & 8.44 & 8.36 & 6.21 & 8.78 & 6.51 & 0.0681 & 9.91 \\
\hline
\end{tabular}




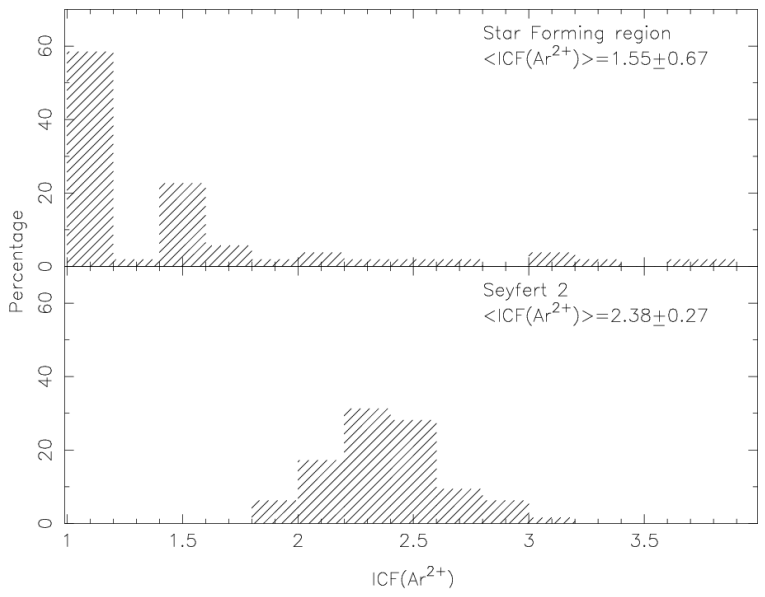

Figure 7. Bottom panel: distribution of the ICF values for the argon from our sample of Seyfert 2 (see Sect. 2.1) derived by using Eqs. 13 and 14. The averaged value is indicated. Top panel: Same as the bottom panel but for ICFs of SFs ( $\mathrm{H}_{\text {II }}$ regions and H I galaxies) derived by Berg et al. (2020, 2015), Croxall et al. (2016, 2015), Bresolin et al. (2009), Hägele et al. (2008), and Lee \& Skillman (2004).

proposed by Dors et al. (2020a) and the ICF for the argon, proposed in this study, hitherto, is the first one for this class of objects. Thus, it is worthwhile to analyse the derived ICF values for our sample as well as to compare it with other proposed derivations for gaseous nebulae. In Fig. 7, bottom panel, the distribution of the argon ICF values derived from our sample through Eq. 13 is shown. We derived ICF values ranging from $\sim 1.8$ to $\sim 3$, with an averaged value of $2.38 \pm 0.27$. Also in Fig. 7, top panel, the argon ICF distribution for $53 \mathrm{SFs}$ (49 disk $\mathrm{H}$ II regions and $4 \mathrm{H}$ II galaxies) compiled from the literature is shown. We notice that majority of SFs present lower ICFs in comparison with those derived from our sample of Seyfert 2 nuclei, implying that $\sim 90 \%$ of the SFs have $\mathrm{ICF}\left(\mathrm{Ar}^{2+}\right) \lesssim 2$ and an average value of about 1.5. These results point to the known fact that the gas phase in Seyfert 2 has a higher excitation degree than SFs.

Regarding abundance estimates, our results for Seyfert 2 nuclei can be used to verify a possible secondary stellar production of the argon at the very high metallicity regime $[12+\log (\mathrm{O} / \mathrm{H}) \gtrsim 8.8]$, because the maximum value derived in most part of SFs through the $T_{\mathrm{e}}$-method is in the order of $12+\log (\mathrm{O} / \mathrm{H}) \sim 8.7$ (e.g. Kennicutt et al. 2003; Berg et al. 2020; Yates et al. 2020). In Fig. 8, the total argon abundance [in units of $12+\log (\mathrm{Ar} / \mathrm{H})$ ] versus the oxygen abundance [in units of $12+\log (\mathrm{O} / \mathrm{H})$ ] from our sample of Seyfert 2 nuclei is shown. Also in this figure, abundance results based on $T_{\mathrm{e}}$-method for galaxy nuclei with star formation derived by Izotov et al. (2006) (whose observational data were also taken from SDSS; Abazajian et al. 2005) as well as results for $\mathrm{H}$ II galaxies obtained by Hägele et al. (2008) are shown. In this case, we chose to compare our results only with those from star-forming galaxies (excluding disk $\mathrm{H}$ in regions) because SFs are subject to similar physical processes like those in AGNs. The following are few examples which underscore such similarities.

(i) SFs and AGNs can present gas outflows (e.g. Hopkins

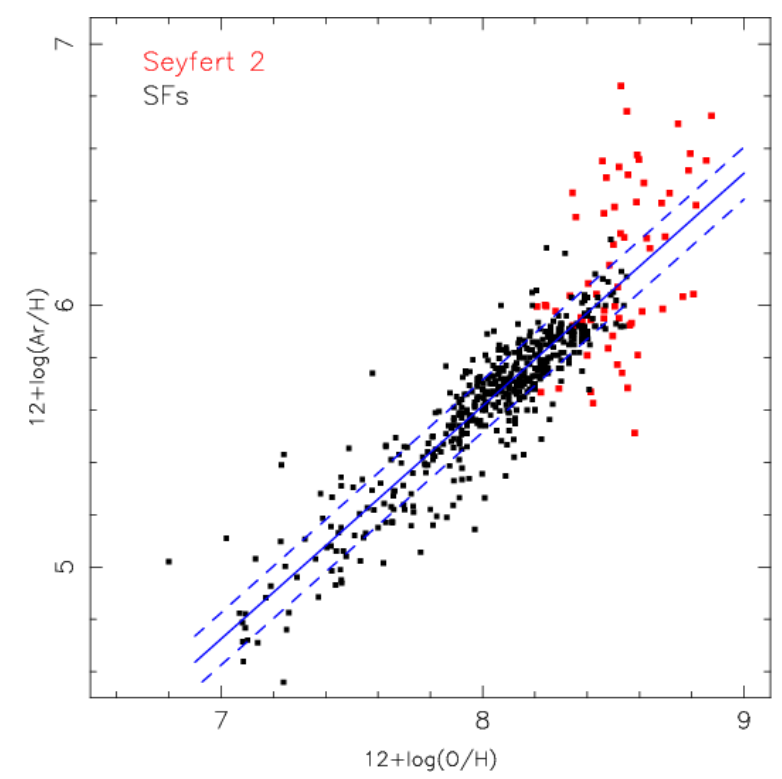

Figure 8. Total argon abundance [in units of $12+\log (\mathrm{Ar} / \mathrm{H})$ ] versus the oxygen abundance [in units of $12+\log (\mathrm{O} / \mathrm{H})]$. Red points represent abundance results from our sample of Seyfert 2. Black points represent abundance results from $\mathrm{H}_{\text {II }}$ galaxies obtained through $T_{\mathrm{e}}$-method by Izotov et al. (2006) and Hägele et al. (2008). The solid line represents a fit to the points (Eq. 16) while the dashed lines represent the uncertainty of \pm 0.1 dex in $\mathrm{H}_{\text {II }}$ region abundance estimations (e.g. Kennicutt et al. 2003).

et al. 2012; Hirschmann et al. 2013; Chisholm et al. 2017; Riffel et al. 2014, 2021; Riffel 2021; Couto et al. 2021).

(ii) In the case of spiral galaxies the presence of bars might produce a falling of gas into the central regions in both objects (e.g. Athanassoula 1992).

These processes can regulate the star formation as well as modify the gas enrichment (e.g. Oppenheimer et al. 2010; Krumholz et al. 2017; Su et al. 2017; James et al. 2020) and could not be present in isolated H II regions. From Fig. 8, we observe a clear linear relation between $\mathrm{Ar} / \mathrm{H}$ and $\mathrm{O} / \mathrm{H}$ indicating a primary production of the argon in a wide metallicity regime. A fit to the points (considering Seyfert 2 and $\mathrm{SFs}$ ) produces the expression

$12+\log (\mathrm{Ar} / \mathrm{H})=[(0.89 \pm 0.01) \times 12+\log (\mathrm{O} / \mathrm{H})]-(1.50 \pm 0.15)$,

represented in Fig. 8 by a solid blue line. The dashed lines in this figure represent the uncertainty of \pm 0.1 dex in abundance estimations (e.g. Kennicutt et al. 2003). It is noteworthy to point out from Fig. 8 that, for very high $\mathrm{O} / \mathrm{H}$ values, a slight deviation of the points representing AGNs results from Eq. 16. This can be an indication of a secondary argon production at very high metallicity regime. In view of this, we tested several polynomial fittings considering different limits for oxygen abundance looking for a transition from primary to secondary Ar production, but no satisfactory solution was obtained. Therefore, we emphasize that our results obtained from AGNs, when combined with those from star-forming galaxies, indicate that the argon has a primary stellar production in a wide metallicity range.

The heavy elements in AGNs are mainly produced by 
stellar evolution in the ISM located at few kpc away from the nuclei (e.g. Boer \& Schulz 1993; Elmegreen et al. 2002; Díaz et al. 2007; Dors et al. 2008; Böker et al. 2008; Riffel et al. 2009; Hägele et al. 2013; Álvarez-Álvarez et al. 2015; Riffel et al. 2016; Pilyugin et al. 2020; Ma et al. 2021) and then transported to the supermassive black hole. Additional metal enrichment by stars can also be obtained by two ways:

(i) in situ star formation embedded in the thin AGN accretion disk (e.g. Collin \& Zahn 1999; Goodman \& Tan 2004; Collin \& Zahn 2008; Wang et al. 2011; Mapelli et al. 2012; Davies \& Lin 2020; Cantiello et al. 2021) and by

(ii) capture of stars orbiting the central regions of galaxies (e.g. Syer et al. 1991; Artymowicz et al. 1993).

Both processes can produce different chemical evolution of AGNs in comparison with that of SFs. For instance, the maximum oxygen abundance in the centres of most luminous star-forming galaxies has been found to be $12+\log (\mathrm{O} / \mathrm{H}) \sim$ 8.9 (e.g. Pilyugin et al. 2007) while Seyfert 2 nuclei can reach up to $\sim 9.2$ dex (e.g. Dors et al. 2020a; Dors 2021), i.e. there is an additional enrichment in Seyferts in comparison with metal rich H II regions. Moreover, recently, Armah et al. (2021) found that $\mathrm{Ne} / \mathrm{H}$ abundance in a small sample of Seyfert $2 \mathrm{~s}$ is nearly 2 times higher than those in SFs. Thus, determining metal abundance in AGNs, in this case argon abundance, can produce constraints in studies on star nucleosynthesis in the very high metallicity regime and in different boundary conditions than those in SFs.

As a result, it is important to verify the range of $\mathrm{Ar} / \mathrm{H}$ abundance in AGNs, since these objects present, in general, higher metallicity (or $\mathrm{O} / \mathrm{H}$ abundance) than star-forming regions (e.g. Groves et al. 2006; Dors et al. 2020a). In Fig. 9, the distributions of argon abundance [in units of $12+\log (\mathrm{Ar} / \mathrm{H})]$ and $\log (\mathrm{Ar} / \mathrm{O})$ for our sample and the solar value for these abundances are shown. In Table 2 the minimum, maximum and the mean abundance values, taken from Fig. 9, as well as the oxygen abundance values (not shown in Fig. 9) are listed. We also listed in Table 2 the minimum, maximum and mean abundance values $\left(W_{\mathrm{v}}\right)$ in relation to the corresponding solar value, defined by

$W_{\mathrm{v}}^{\mathrm{X}}=\mathrm{X}(\mathrm{v}) / \mathrm{X}_{\odot}$,

where $\mathrm{X}$ is a given abundance ratio. The solar argon and oxygen abundances are those derived by Grevesse \& Sauval (1998) and Allende Prieto et al. (2001), respectively. It can be inferred from Table 2 that the maximum $\mathrm{Ar} / \mathrm{H}$ and $\mathrm{Ar} / \mathrm{O}$ abundance ratios in our sample of AGNs are higher than the solar values, i.e. $\sim 2.7$ and $\sim 4.0$ times the solar values, respectively. However, the $W_{\max }^{\mathrm{Ar} / \mathrm{H}}$ and $W_{\max }^{\mathrm{Ar} / \mathrm{O}}$ can even be higher than the ones found in this work due to the sample considered. Actually, the $W_{\max }^{\mathrm{O} / \mathrm{H}}$ derived for our sample is about 1.5 times the solar value. Dors et al. (2020a) found $W_{\text {max. }}^{\mathrm{O} / \mathrm{H}} \sim 3.2$ after considering a larger sample of SDSS Seyfert 2 nuclei without adopting the [Ar III] emission line presence as a selection criterion. Thus, this disparity indicates the probable existence of oversolar $\mathrm{Ar} / \mathrm{H}$ and $\mathrm{Ar} / \mathrm{O}$ values higher than those listed in Table 2 .

In order to compare the abundance range of $\mathrm{Ar} / \mathrm{H}$ and $\mathrm{Ar} / \mathrm{O}$ derived through the new methodology from the $T_{\mathrm{e}^{-}}$ method for AGNs with those obtained from other methods, we compiled from the literature $\mathrm{Ar} / \mathrm{H}$ and $\mathrm{Ar} / \mathrm{O}$ radial gradients derived in spiral galaxies and calculated by the stan-

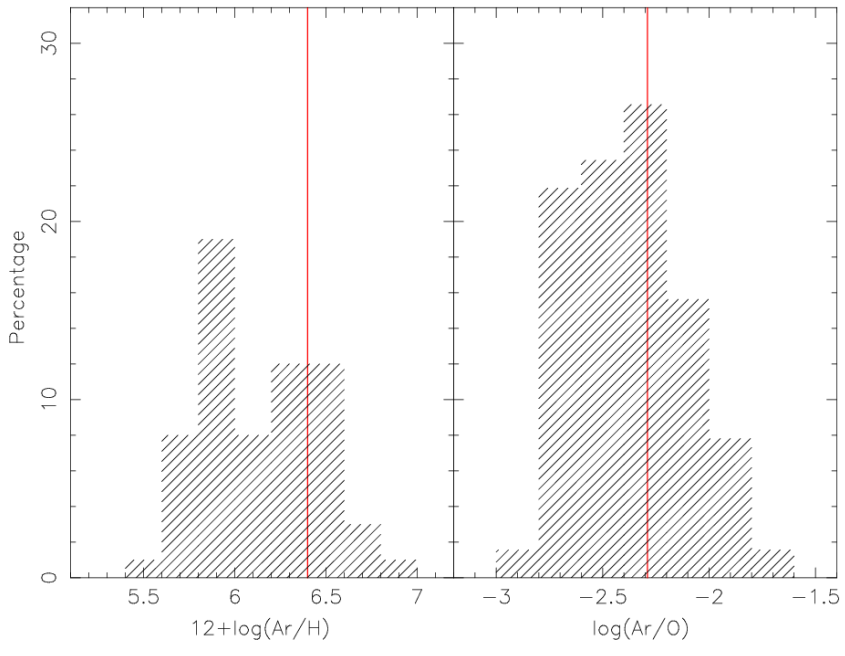

Figure 9. Left panel: distribution of argon abundance [in units of $12+\log (\mathrm{Ar} / \mathrm{H})]$ for our sample of Seyfert 2 nuclei. The red line represents the solar argon abundance $\left[12+\log (\mathrm{Ar} / \mathrm{H})_{\odot}=6.40\right]$ derived by Grevesse \& Sauval (1998). Right panel: same as the left panel but for $\log (\mathrm{Ar} / \mathrm{O})$. The red line represent the solar argon abundance $\left[\log (\mathrm{Ar} / \mathrm{O})_{\odot}=-2.29\right]$ derived by Grevesse \& Sauval (1998) and Allende Prieto et al. (2001).

dard $T_{\mathrm{e}}$-method. We extrapolated these radial gradients to the central part of the galaxies (i.e. galactocentric distance $R=0)$. This methodology makes it possible to infer indirect and independent values of abundances in the nuclei of spiral galaxies (e.g. Vila-Costas \& Edmunds 1992; van Zee et al. 1998; Pilyugin et al. 2004; Zinchenko et al. 2019). As usual, we assume that the $\mathrm{Ar} / \mathrm{H}$ and $\mathrm{Ar} / \mathrm{O}$ radial abundance gradients are represented by

$Y^{\mathrm{X}}=Y_{0}^{\mathrm{X}}+\left[\operatorname{grad} Y^{\mathrm{X}} \times R(\mathrm{kpc})\right]$,

where $Y^{\mathrm{X}}$ represents the value of the abundance ratio $\mathrm{X}$ $[12+\log (\mathrm{Ar} / \mathrm{H})$ or $\log (\mathrm{Ar} / \mathrm{O})], Y_{0}^{\mathrm{X}}$ is the extrapolated value of $\mathrm{X}$ to the galactic center, i.e. at radial distance $R=0$, and $\operatorname{grad} Y^{\mathrm{X}}$ is the slope of the distribution expressed in units of dex kpc ${ }^{-1}$. As pointed out by Pilyugin et al. (2004), the accuracy of radial abundance gradient determinations is defined not only by the large number of objects considered but also by the distribution of these objects along the galactic radius. Under this supposition, we take into consideration published data from the literature for $\mathrm{X}$ abundance values of $\mathrm{H}_{\mathrm{II}}$ regions derived by using the $T_{\mathrm{e}}$-method and located at galactocentric distances in spiral galaxies within the range $0.2 \lesssim\left(R / R_{25}\right) \lesssim 1$, where $R$ is the galactocentric distance and $R_{25}$ is the $B$-band isophote at a surface brightness of $25 \mathrm{mag} \operatorname{arcsec}^{-2}$. It was possible to obtain the $\mathrm{Ar} / \mathrm{H}$ and $\mathrm{Ar} / \mathrm{O}$ radial gradients in four spiral galaxies. In Table 3, the identification of each galaxy, the number $(N)$ of $\mathrm{H}$ II regions considered in deriving the radial gradients, the $Y_{0}{ }^{\mathrm{X}}$ and $\operatorname{grad} Y^{\mathrm{X}}$ values as well as references to the original studies from which the data were obtained are listed. Also in Table 3 , the $Y_{0}^{\mathrm{X}}$ in relation to the solar value, defined by $W_{0}^{X}=Y_{0}^{\mathrm{X}} /(\mathrm{X}) \odot$

is listed. In Fig. 10, the $W_{0}^{\mathrm{X}}$ values for the four spiral galaxies, listed in Table 3, are represented by red points and compared with those for the range of values (hatched areas) de- 
Table 2. Minimum, maximum and the mean abundance ratio values for our sample (see Sect. 2.1) derived through $T_{\mathrm{e}}$-method adapted for AGNs (see Sect. 2.2). The abundance values are obtained from the distributions presented in Fig. 9. The $W_{\min }^{\mathrm{X}}, W_{\max }^{\mathrm{X}}$ and $W_{\text {mean }}^{\mathrm{X}}$ are calculated from Eq. 17 and represent abundance values relative to the solar values. The solar argon and oxygen abundances considered were those derived by Grevesse \& Sauval (1998) and Allende Prieto et al. (2001).

\begin{tabular}{lrrrrrr}
\hline Abundance ratio & Min. & Max. & Mean & $W_{\text {min. }}^{\mathrm{X}}$ & $W_{\text {max. }}^{\mathrm{X}}$ & $W_{\text {mean }}^{\mathrm{X}}$ \\
\hline $12+\log (\mathrm{Ar} / \mathrm{H})$ & 5.51 & 6.84 & $6.14 \pm 0.32$ & 0.13 & 2.75 & $0.54_{-0.27}^{+0.60}$ \\
$\log (\mathrm{Ar} / \mathrm{O})$ & -3.06 & -1.68 & $-2.37 \pm 0.28$ & 0.16 & 3.98 & $0.97_{-0.53}^{+0.61}$ \\
$12+\log (\mathrm{O} / \mathrm{H})$ & 8.20 & 8.87 & $8.52 \pm 0.16$ & 0.30 & 1.51 & $0.67_{-0.20}^{+0.30}$ \\
\hline
\end{tabular}

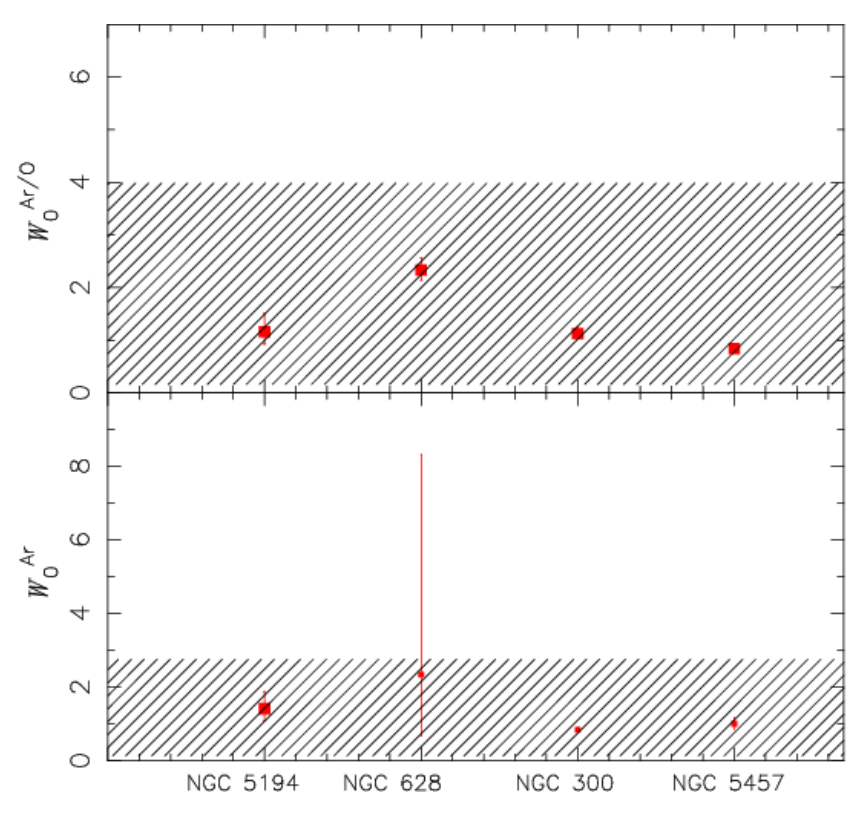

Figure 10. Comparison between argon abundance in relation to the solar value for $W_{0}^{\mathrm{Ar}}=(\mathrm{Ar}) /(\mathrm{Ar}) \odot($ bottom panel $)$ and $W_{0}^{\mathrm{Ar} / \mathrm{O}}=(\mathrm{Ar} / \mathrm{O}) /(\mathrm{Ar} / \mathrm{O}) \odot($ top panel $)$. Points represent estimates from radial abundance extrapolations to the central parts (galactocentric distance $R=0$ ) from the four spiral galaxies listed in Table 3 and indicated in the $\mathrm{x}$-axis

. The hatched areas represent the range of abundance ratio values derived for our sample of Seyfert 2 listed in Table 2.

rived for our sample and listed in Table 2. It can be seen that our range of abundance estimates are in consonance with the values obtained by independent abundance estimates from the radial gradients. Obviously, a more exact comparison would be obtained if both $\mathrm{Ar} / \mathrm{H}$ and $\mathrm{O} / \mathrm{H}$ abundances in the AGNs and the radial abundances of these elements in the host galaxies were determined, such as studies carried out for the oxygen by Storchi-Bergmann et al. (1998b) and Dors et al. (2015).

Finally, in Fig. 11, our Seyfert 2 estimates (red points) for $(\mathrm{Ar} / \mathrm{O})$ versus $(\mathrm{O} / \mathrm{H})$ abundances and those calculated through the $T_{\mathrm{e}}$-method for star-forming nuclei by Izotov et al. (2006) and H II galaxies by Hägele et al. (2008) are shown. As for the neon and sulphur (see Dors et al. 2013, 2016 and references therein) several studies on $(\mathrm{Ar} / \mathrm{O})$ versus $(\mathrm{O} / \mathrm{H})$ have yielded conflicting results. For instance, Izotov et al. (2006), by correcting the depletion of oxygen onto dust grains (which is in order of $\sim 0.1 \mathrm{dex}$ ) found a negative slope for $\mathrm{Ar} / \mathrm{O}$ versus $\mathrm{O} / \mathrm{H}$ for the range $7.1 \lesssim[12+\log (\mathrm{O} / \mathrm{H})] \lesssim 8.6$, which correspond to the metallicity range $0.03 \lesssim\left(\mathrm{Z} / \mathrm{Z}_{\odot}\right) \lesssim 0.7$. This negative slope is strongly influenced by estimations in objects with very low metallicity $[12+\log (\mathrm{O} / \mathrm{H})] \lesssim 7.5]$, in which oversolar $\mathrm{Ar} / \mathrm{O}$ abundance values are derived. Although this result of a negative slope is in consonance with the one found by Pérez-Montero et al. (2007), who considered H II galaxies and H II regions estimates, these authors derived solar $\mathrm{Ar} / \mathrm{O}$ abundance ratios for objects at low metallicity regime and subsolar values for the ones at high metallicity regime. Finally, Berg et al. (2020), who took into account the $T_{\mathrm{e}}$-method abundance estimates for 190 disk $\mathrm{H}$ II regions, derived sub-solar $\mathrm{Ar} / \mathrm{O}$ abundance ratio for some few $\mathrm{H}$ in regions with low metallicity $[12+\log (\mathrm{O} / \mathrm{H}) \lesssim 8.0]$. In principle, a possible explanation for the discrepancies above is the use of different argon ICFs by the authors rather than the consideration of distinct samples. In any case, it can be seen from Fig. 11 that, a slight decrease of $\mathrm{Ar} / \mathrm{O}$ with the increase of $\mathrm{O} / \mathrm{H}$, when our estimates are combined with those for $\mathrm{H}$ II galaxies. It is worth to mention that, for $[12+\log (\mathrm{O} / \mathrm{H}) \gtrsim 8.0]$ our $\mathrm{Ar} / \mathrm{O}$ estimates are in consonance with those derived for $\mathrm{H}$ II galaxies. A linear regression to the points produces

$\log (\mathrm{Ar} / \mathrm{O})=(-0.11 \pm 0.02) \mathrm{x}-(1.51 \pm 0.15)$,

where $\mathrm{x}=\log (\mathrm{O} / \mathrm{H})$.

\section{CONCLUSIONS}

For the fist time, quantitative argon abundances, based on $T_{\mathrm{e}}$-method adapted for AGNs, is derived for the narrow regions of Seyfert 2 nuclei. In view of this, we compiled from Sloan Digital Sky Survey Data Release 7 (SDSS-DR7) optical narrow emission line fluxes for 64 Seyfert 2 galaxies in the local universe $(z<0.25)$ and calculated the abundance of the argon and oxygen in relation to hydrogen $(\mathrm{Ar} / \mathrm{H}, \mathrm{O} / \mathrm{H})$. The total argon abundance relative to hydrogen $(\mathrm{Ar} / \mathrm{H})$ was derived for each object of the sample through the $\mathrm{Ar}^{2+} / \mathrm{H}^{+}$ionic abundance and by using a theoretical expression for the Ionization Correction Factor (ICF) obtained from photoionization model results built with the CLOUDY code. These results from the models were also used to derive an appropriate temperature for the $\mathrm{Ar}^{2+}$ $\left[T_{\mathrm{e}}(\mathrm{Ar}\right.$ III $\left.)\right]$ which can be derived by its dependence on the temperature for $\mathrm{O}^{2+}\left[T_{\mathrm{e}}(\mathrm{O}\right.$ III $\left.)\right]$ calculated by using the observational $[\mathrm{O}$ III $](\lambda 4959+\lambda 5007) / \lambda 4363$ line ratio. We obtained the following conclusions:

(i) The equality between the temperatures 
Table 3. Parameters of the radial abundance gradients derived for the $\mathrm{Ar} / \mathrm{H}$ and $\mathrm{Ar} / \mathrm{O}$ abundance ratios in a sample of spiral galaxies. $N$ represents the number of $\mathrm{H}_{\mathrm{II}}$ regions considered in the estimations of the gradients. $Y_{0}, \operatorname{grad} Y$ and $W_{0}$ are defined in Eqs. 18 and 19. In the last column, the original works from which the radial gradients were compiled are listed.

\begin{tabular}{lcrrrrrrr}
\hline Object & $N$ & $Y_{0} \mathrm{Ar} / \mathrm{H}$ & $\operatorname{grad} Y^{\mathrm{Ar} / \mathrm{H}}$ & $W_{0}^{\mathrm{Ar}}$ & $Y_{0} \mathrm{Ar} / \mathrm{O}$ & $\operatorname{grad} Y^{\mathrm{Ar} / \mathrm{O}}$ & $W_{0}^{\mathrm{Ar} / \mathrm{O}}$ & Reference \\
\hline NGC 5194 & 28 & $6.55 \pm 0.12$ & $-0.015 \pm 0.026$ & $1.41_{-0.33}^{+0.45}$ & $-2.22 \pm 0.11$ & $+0.013 \pm 0.023$ & $1.16_{-0.24}^{+0.35}$ & Croxall et al. (2015) \\
NGC 628 & 45 & $6.77 \pm 0.55$ & $-0.070 \pm 0.009$ & $2.34_{-1.66}^{+5.97}$ & $-1.92 \pm 0.04$ & $-0.037 \pm 0.006$ & $2.33_{-0.19}^{+0.24}$ & Berg et al. $(2015)$ \\
NGC 300 & 28 & $6.32 \pm 0.03$ & $-0.106 \pm 0.011$ & $0.84_{-0.03}^{+0.05}$ & $-2.24 \pm 0.03$ & $-0.028 \pm 0.009$ & $1.12_{-0.07}^{+0.12}$ & Bresolin et al. (2009) \\
NGC 5457 & 16 & $6.40 \pm 0.07$ & $-0.020 \pm 0.003$ & $1.00_{-0.14}^{+0.17}$ & $-2.36 \pm 0.01$ & $-0.008 \pm 0.002$ & $0.84_{-0.01}^{+0.03}$ Kennicutt et al. (2003) \\
\hline
\end{tabular}

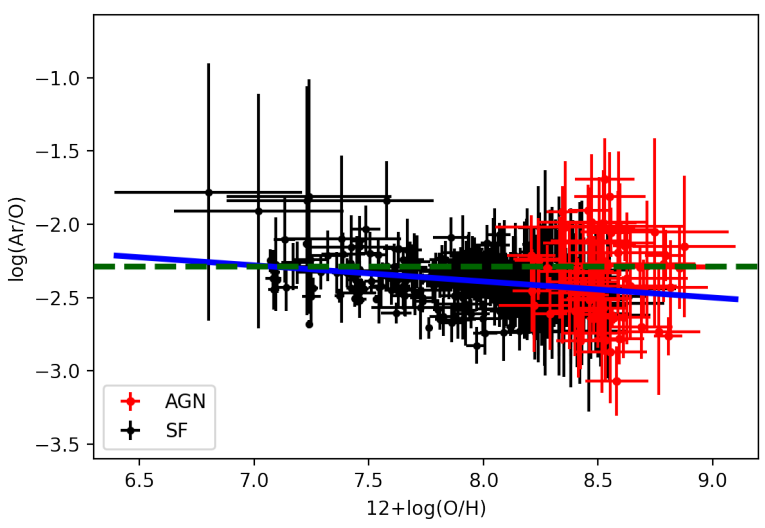

Figure 11. Relation between $\log (\mathrm{Ar} / \mathrm{O})$ and $12+\log (\mathrm{O} / \mathrm{H})$. Red points represent estimations for our sample of Seyfert 2 while black points represent compiled estimations for $\mathrm{H}$ II galaxies derived by Izotov et al. (2006) and Hägele et al. (2008). The blue solid line represents the linear regression to the points given by Eq. 20. The dashed green line represents the solar value, $\log (\mathrm{Ar} / \mathrm{O})_{\odot}=-2.29$, derived by Grevesse \& Sauval (1998) and Allende Prieto et al. (2001).

$T_{\mathrm{e}}(\mathrm{S}$ III $)=T_{\mathrm{e}}($ Ar III $)$, usually assumed in abundance studies of star-forming regions, is not valid for Seyfert 2 since the nebular gas region occupied by $\mathrm{Ar}^{2+}$ tends to have a lower temperature than the nebular gas region occupied by $\mathrm{S}^{2+}$.

(ii) A bi-parametric expression for the $\operatorname{ICF}\left(\mathrm{Ar}^{2+}\right)$ as function of the $\mathrm{x}=\left[\mathrm{O}^{2+} /\left(\mathrm{O}^{+}+\mathrm{O}^{2+}\right)\right]$ abundance ratio and the oxygen abundance [in units of $12+\log (\mathrm{O} / \mathrm{H})]$ is proposed to derive the total argon abundance.

(iii) For the range of oxygen abundance $8.0 \lesssim[12+$ $\log (\mathrm{O} / \mathrm{H})] \lesssim 9.0$ or metallicity $0.20 \lesssim\left(Z / \mathrm{Z}_{\odot}\right) \lesssim 2.0$, we found that our sample of Seyfert 2 present $\mathrm{A} / \mathrm{H}$ abundances ranging from $\sim 0.1$ to $\sim 3$ times the argon solar value, indicating that most of the objects $(\sim 75 \%)$ have subsolar argon abundance.

(iv) The range of $\mathrm{Ar} / \mathrm{H}$ and $\mathrm{Ar} / \mathrm{O}$ abundance values obtained for our sample are in consonance with those estimated from extrapolations to the central parts of radial abundance gradients derived in the disk of four spiral galaxies. (v) We found a slight tendency of $\mathrm{Ar} / \mathrm{O}$ abundance ratio decreases with $\mathrm{O} / \mathrm{H}$.

(vi) Finally, the Ar/O abundance values for our sample of Seyfert 2 are in consonance with estimations for $\mathrm{H}$ in galaxies $[12+\log (\mathrm{O} / \mathrm{H}) \gtrsim 8.0]$, indicating that there is not an over enrichment of argon in AGNs, at least for the metallicity range considered.

\section{ACKNOWLEDGEMENTS}

AFM gratefully acknowledges support from Coordenação de Aperfeiçoamento de Pessoal de Nível Superior (CAPES). OLD is grateful to Fundação de Amparo à Pesquisa do Estado de São Paulo (FAPESP) and Conselho Nacional de Desenvolvimento Científico e Tecnológico (CNPq).

\section{DATA AVAILABILITY}

The data underlying this article will be shared on reasonable request with the corresponding author.

\section{REFERENCES}

Abazajian K., et al., 2005, AJ, 129, 1755

Alexander J., Balick B., 1997, AJ, 114, 713

Allende Prieto C., Lambert D. L., Asplund M., 2001, ApJ, 556, L63

Alloin D., Bica E., Bonatto C., Prugniel P., 1992, A\&A, 266, 117

Álvarez-Álvarez M., Díaz A. I., Terlevich E., Terlevich R., 2015, MNRAS, 451, 3173

Amayo A., Delgado-Inglada G., Stasińska G., 2021, MNRAS, 505, 2361

Armah M., et al., 2021, arXiv e-prints, p. arXiv:2109.04596

Artymowicz P., Lin D. N. C., Wampler E. J., 1993, ApJ, 409, 592

Athanassoula E., 1992, MNRAS, 259, 345

Baldwin J. A., Phillips M. M., Terlevich R., 1981, PASP, 93, 5

Bastian N., Covey K. R., Meyer M. R., 2010, ARA\&A, 48, 339

Berg D. A., Skillman E. D., Croxall K. V., Pogge R. W., Moustakas J., Johnson-Groh M., 2015, ApJ, 806, 16

Berg D. A., Pogge R. W., Skillman E. D., Croxall K. V., Moustakas J., Rogers N. S. J., Sun J., 2020, ApJ, 893, 96

Boer B., Schulz H., 1993, A\&A, 277, 397

Böker T., Falcón-Barroso J., Schinnerer E., Knapen J. H., Ryder S., 2008, AJ, 135, 479

Bresolin F., Gieren W., Kudritzki R.-P., Pietrzyński G., Urbaneja M. A., Carraro G., 2009, ApJ, 700, 309

Cantiello M., Jermyn A. S., Lin D. N. C., 2021, ApJ, 910, 94

Carvalho S. P., et al., 2020, MNRAS, 000 
Castro C. S., Dors O. L., Cardaci M. V., Hägele G. F., 2017, MNRAS, 467, stx150

Chisholm J., Tremonti C. A., Leitherer C., Chen Y., 2017, MNRAS, 469, 4831

Collin S., Zahn J.-P., 1999, Ap\&SS, 265, 501

Collin S., Zahn J. P., 2008, A\&A, 477, 419

Congiu E., et al., 2017, MNRAS, 471, 562

Contini M., 2017, MNRAS, 469, 3125

Couto G. S., Hughes T. M., Boquien M., Ibar E., Viaene S., Leiton R., Xue Y., 2021, arXiv e-prints, p. arXiv:2108.02334

Croxall K. V., Pogge R. W., Berg D. A., Skillman E. D., Moustakas J., 2015, ApJ, 808, 42

Croxall K. V., Pogge R. W., Berg D. A., Skillman E. D., Moustakas J., 2016, ApJ, 830, 4

Davies M. B., Lin D. N. C., 2020, MNRAS, 498, 3452

Delgado-Inglada G., Morisset C., Stasińska G., 2014, MNRAS, 440,536

Díaz Á. I., Terlevich E., Castellanos M., Hägele G. F., 2007, MNRAS, 382, 251

Dopita M. A., et al., 2015, ApJS, 217, 12

Dors O., 2021, arXiv e-prints, p. arXiv:2107.11606

Dors O. L. J., Storchi-Bergmann T., Riffel R. A., Schimdt A. A., 2008, A\&A, 482, 59

Dors O. L., et al., 2013, MNRAS, 432, 2512

Dors O. L., Cardaci M. V., Hägele G. F., Rodrigues I., Grebel E. K., Pilyugin L. S., Freitas-Lemes P., Krabbe A. C., 2015, MNRAS, 453, 4103

Dors O. L., Pérez-Montero E., Hägele G. F., Cardaci M. V., Krabbe A. C., 2016, MNRAS, 456, 4407

Dors O. L., Arellano-Córdova K. Z., Cardaci M. V., Hägele G. F., 2017, MNRAS: Letters, 468, L113

Dors O. L., Maiolino R., Cardaci M. V., Hägele G. F., Krabbe A. C., Pérez-Montero E., Armah M., 2020a, MNRAS, 14, 1

Dors O. L., et al., 2020b, MNRAS, 492, 468

Dors O. L., Contini M., Riffel R. A., Pérez-Montero E., Krabbe A. C., Cardaci M. V., Hägele G. F., 2021, MNRAS, 501, 1370

Elmegreen D. M., Chromey F. R., McGrath E. J., Ostenson J. M., 2002, AJ, 123, 1381

Feltre A., Charlot S., Gutkin J., 2016, MNRAS, 456, 3354

Ferland G. J., Osterbrock D. E., 1986, ApJ, 300, 658

Ferland G. J., et al., 2013, RMAA, 49, 137

Ferland G. J., et al., 2017, RMxAA, 53, 385

Flury S. R., Moran E. C., 2020, MNRAS, 496, 2191

French H. B., 1981, ApJ, 246, 434

Garnett D. R., 1992, AJ, 103, 1330

Goodman J., Tan J. C., 2004, ApJ, 608, 108

Grevesse N., Sauval A. J., 1998, Space Sci. Rev., 85, 161

Groves B. A., Heckman T. M., Kauffmann G., 2006, MNRAS, 371,1559

Guseva N. G., Izotov Y. I., Stasińska G., Fricke K. J., Henkel C., Papaderos P., 2011, A\&A, 529, A149

Hägele G. F., Díaz Á. I., Terlevich E., Terlevich R., Pérez-Montero E., Cardaci M. V., 2008, MNRAS, 383, 209

Hägele G. F., Díaz Á. I., Terlevich R., Terlevich E., Bosch G. L., Cardaci M. V., 2013, MNRAS, 432, 810

Henry R. B. C., Kwitter K. B., Howard J. W., 1996, ApJ, 458, 215

Hirschmann M., et al., 2013, MNRAS, 436, 2929

Hopkins P. F., Kereš D., Murray N., Quataert E., Hernquist L., 2012, MNRAS, 427, 968

Izotov Y. I., Thuan T. X., 1999, ApJ, 511, 639

Izotov Y. I., Thuan T. X., 2008, ApJ, 687, 133

Izotov Y. I., Stasińska G., Meynet G., Guseva N. G., Thuan T. X., 2006, A\&A, 448, 955

James B. L., Kumari N., Emerick A., Koposov S. E., McQuinn K. B. W., Stark D. P., Belokurov V., Maiolino R., 2020, MNRAS, 495,2564

Kennicutt R. C., Bresolin F., Garnett D. R., 2003, ApJ, 591, 801
Kewley L. J., Dopita M. A., Sutherland R. S., Heisler C. A., Trevena J., 2001, ApJ, 556, 121

Kewley L. J., Nicholls D. C., Sutherland R. S., 2019, ARA\&A, 57,511

Koski A. T., 1978, ApJ, 223, 56

Krabbe A. C., Oliveira C. B., Zinchenko I. A., Hernández-Jiménez J. A., Dors O. L., Hägele G. F., Cardaci M. V., Telles N. R., 2021, MNRAS, 505, 2087

Krumholz M. R., Kruijssen J. M. D., Crocker R. M., 2017, MNRAS, 466, 1213

Lee H., Skillman E. D., 2004, ApJ, 614, 698

Luridiana V., Morisset C., Shaw R. A., 2015, A\&A, 573

Ma J., Maksym W. P., Fabbiano G., Elvis M., Storchi-Bergmann T., Karovska M., Wang J., Travascio A., 2021, ApJ, 908, 155

Maciel W. J., Quireza C., Costa R. D. D., 2007, A\&A, 463, L13

Mapelli M., Hayfield T., Mayer L., Wadsley J., 2012, ApJ, 749, 168

Mathis J. S., 1985, ApJ, 291, 247

McGaugh S. S., 1991, ApJ, 380, 140

Morisset C., 2018, in Hägele G., Cardaci M., Pérez-Montero E., eds, Chemical Abundances in Gaseous Nebulae. p. 33

Morisset C., et al., 2016, A\&A, 594, A37

Oppenheimer B. D., Davé R., Kereš D., Fardal M., Katz N., Kollmeier J. A., Weinberg D. H., 2010, MNRAS, 406, 2325

Osterbrock D. E., Miller J. S., 1975, ApJ, 197, 535

Pagomenos G. J. S., Bernard-Salas J., Pottasch S. R., 2018, A\&A, 615, A29

Peimbert M., Costero R., 1969, Boletin de los Observatorios Tonantzintla y Tacubaya, 5,3

Peimbert M., Peimbert A., Delgado-Inglada G., 2017, PASP, 129, 082001

Pérez-Díaz B., Masegosa J., Márquez I., Pérez-Montero E., 2021, MNRAS, 505, 4289

Pérez-Montero E., 2017, PASP, 129, 043001

Pérez-Montero E., Díaz Á. I., 2007, MNRAS, 377, 1195

Pérez-Montero E., Hägele G. F., Contini T., Díaz Á. I., 2007, MNRAS, 381, 125

Pérez-Montero E., García-Benito R., Hägele G. F., Díaz Á. I., 2010, MNRAS, 404, 2037

Pérez-Montero E., Dors O. L., Vílchez J. M., García-Benito R., Cardaci M. V., Hägele G. F., 2019, MNRAS, 489, 2652

Pilyugin L. S., 2003, A\&A, 399, 1003

Pilyugin L. S., Vílchez J. M., Contini T., 2004, A\&A, 425, 849

Pilyugin L. S., Thuan T. X., Vílchez J. M., 2007, MNRAS, 376, 353

Pilyugin L. S., Grebel E. K., Zinchenko I. A., Lara-López M. A., Nefedyev Y. A., Shulga V. M., 2020, A\&A, 639, A96

Polles F. L., et al., 2019, A\&A, 622, A119

Revalski M., Crenshaw D. M., Kraemer S. B., Fischer T. C., Schmitt H. R., Machuca C., 2018a, ApJ, 856, 46

Revalski M., et al., 2018b, ApJ, 867, 88

Revalski M., et al., 2021, ApJ, 910, 139

Riffel R. A., 2021, MNRAS, 506, 2950

Riffel R. A., Storchi-Bergmann T., Dors O. L., Winge C., 2009, MNRAS, 393, 783

Riffel R. A., Storchi-Bergmann T., Riffel R., 2014, ApJ, 780, L24

Riffel R. A., et al., 2016, MNRAS, 461, 4192

Riffel R. A., et al., 2021, MNRAS, 501, L54

Stasińska G., 1984, A\&A, 135, 341

Stasińska G., 2002, arXiv e-prints, pp astro-ph/0207500

Storchi-Bergmann T., Schmitt H. R., Calzetti D., Kinney A. L., 1998a, AJ, 115, 909

Storchi-Bergmann T., Schmitt H. R., Calzetti D., Kinney A. L., 1998b, AJ, 115, 909

Su K.-Y., Hopkins P. F., Hayward C. C., Faucher-Giguère C.-A., Kereš D., Ma X., Robles V. H., 2017, MNRAS, 471, 144

Syer D., Clarke C. J., Rees M. J., 1991, MNRAS, 250, 505 
Thomas A. D., Kewley L. J., Dopita M. A., Groves B. A., Hopkins A. M., Sutherland R. S., 2019, ApJ, 874, 100

Thuan T. X., Izotov Y. I., Lipovetsky V. A., 1995, ApJ, 445, 108 Toribio San Cipriano L., Domínguez-Guzmán G., Esteban C., García-Rojas J., Mesa-Delgado A., Bresolin F., Rodríguez M., Simón-Díaz S., 2017, MNRAS, 467, 3759

Torres-Peimbert S., Peimbert M., 1977, Rev. Mex. Astron. Astrofis., 2, 181

Vaona Z. T., Trocar Y. C., Hammer F., 2012, MNRAS, 427, 1266

Vila-Costas M. B., Edmunds M. G., 1992, MNRAS, 259, 121

Wang J.-M., et al., 2011, ApJ, 739, 3

Wesson R., Liu X. W., Barlow M. J., 2005, MNRAS, 362, 424

Williams R. E., 1967, ApJ, 147, 556

Woosley S. E., Weaver T. A., 1995, ApJS, 101, 181

Yates R. M., Kauffmann G., Guo Q., 2012, MNRAS, 422, 215

Yates R. M., Schady P., Chen T. W., Schweyer T., Wiseman P., 2020, A\&A, 634, A107

York D. G., Adelman J., Anderson Jr. J. E., Anderson S. F., Annis J., Bahcall 2000, AJ, 120, 1579

Zinchenko I. A., Dors O. L., Hägele G. F., Cardaci M. V., Krabbe A. C., 2019, MNRAS, 483, 1901

Zurita A., Bresolin F., 2012, MNRAS, 427, 1463

van Zee L., Haynes M. P., 2006, ApJ, 636, 214

van Zee L., Salzer J. J., Haynes M. P., O'Donoghue A. A., Balonek T. J., 1998, AJ, 116, 2805

This paper has been typeset from a $\mathrm{T}_{\mathrm{E}} \mathrm{X} / \mathrm{LAT}_{\mathrm{E}} \mathrm{X}$ file prepared by the author. 